\title{
A chemorepulsion model with superlinear production: analysis of the continuous problem and two approximately positive and energy-stable schemes
}

\section{F. Guillén-González ${ }^{1}$ (D) M. A. Rodríguez-Bellido ${ }^{1}$ - D. A. Rueda-Gómez ${ }^{2}$}

Received: 11 October 2020 / Accepted: 12 October 2021 / Published online: 10 December 2021

(C) The Author(s) 2021

\section{Abstract}

We consider the following repulsive-productive chemotaxis model: find $u \geq 0$, the cell density, and $v \geq 0$, the chemical concentration, satisfying

$$
\left\{\begin{array}{l}
\partial_{t} u-\Delta u-\nabla \cdot(u \nabla v)=0 \text { in } \Omega, t>0 \\
\partial_{t} v-\Delta v+v=u^{p} \text { in } \Omega, t>0
\end{array}\right.
$$

with $p \in(1,2), \Omega \subseteq \mathbb{R}^{d}$ a bounded domain ( $\left.d=1,2,3\right)$, endowed with non-flux boundary conditions. By using a regularization technique, we prove the existence of global in time weak solutions of (1) which is regular and unique for $d=1,2$. Moreover, we propose two fully discrete Finite Element (FE) nonlinear schemes, the first one defined in the variables $(u, v)$ under structured meshes, and the second one by using the auxiliary variable $\sigma=\nabla v$ and defined in general meshes. We prove some unconditional properties for both schemes, such as mass-conservation, solvability, energy-stability and approximated positivity. Finally, we compare the behavior of these schemes with respect to the classical FE backward Euler scheme throughout several numerical simulations and give some conclusions.

Keywords Chemorepulsion model · Finite element approximation . Energy-stability $\cdot$ Nonlinear production $\cdot$ Approximated positivity

Mathematics Subject Classification (2010) 35K51 - 35Q92 - 65M12 - 65M60 . $92 \mathrm{C} 17$

\footnotetext{
Communicated by: Jon Wilkening

F. Guillén-González guillen@us.es
}

Extended author information available on the last page of the article. 


\section{Introduction}

Chemotaxis is the biological process of the movement of living organisms in response to a chemical stimulus, movement that can be addressed towards a higher (chemo-attraction) or lower (chemorepulsion) concentration of a chemical substance. At the same time, the presence of living organisms can produce or consume chemical substance. A repulsive-productive chemotaxis model can be given by the following parabolic PDE's system:

$$
\left\{\begin{array}{l}
\partial_{t} u-\Delta u=\nabla \cdot(u \nabla v) \quad \text { in } \Omega, t>0, \\
\partial_{t} v-\Delta v+v=f(u) \quad \text { in } \Omega, t>0
\end{array}\right.
$$

where $u=u(\boldsymbol{x}, t) \geq 0$ and $v=v(\boldsymbol{x}, t) \geq 0$ denote, respectively, the cell density and the concentration of a repulsive chemical signal at position $\boldsymbol{x} \in \Omega \subseteq \mathbb{R}^{d}(d=$ $1,2,3$, being $\Omega$ a bounded domain with boundary $\partial \Omega$ ) and at time $t>0$. Moreover, $f(u) \geq 0$ (if $u \geq 0$ ) is the production term. In this paper, we consider the particular case of superlinear signal production, that is, $f(u)=u^{p}$, with $1<p<2$, and then we focus on the initial-boundary value problem:

$$
\left\{\begin{array}{l}
\partial_{t} u-\Delta u=\nabla \cdot(u \nabla v) \text { in } \Omega, t>0, \\
\partial_{t} v-\Delta v+v=u^{p} \text { in } \Omega, t>0, \\
\frac{\partial u}{\partial \boldsymbol{n}}=\frac{\partial v}{\partial \boldsymbol{n}}=0 \text { on } \partial \Omega, t>0, \\
u(\boldsymbol{x}, 0)=u_{0}(\boldsymbol{x}) \geq 0, v(\boldsymbol{x}, 0)=v_{0}(\boldsymbol{x}) \geq 0 \text { in } \Omega .
\end{array}\right.
$$

From the biological point of view, the nonlinear signal production considered in model (3) is justified and explains the saturation effects of chemotactic signal production at large (or short) densities of cells (see [32] and references therein).

The theoretical analysis of chemorepulsion models has included the study of some qualitative properties of the solutions, such as existence, uniqueness, regularity and behavior at infinite time, among others [9, 14, 17, 30, 31]. In the case of linear $(p=1)$ or quadratic $(p=2)$ production term, problem (3) is well-posed (see [9, 17] respectively) in the following sense: there exist global in time weak solutions in $3 D$ domains, which are regular (and unique) for $1 D$ and $2 D$ domains. In [14], the uniqueness and global existence of solution for a chemorepulsion model with linear production and superlinear diffusion in $d D$ domains (for $d \geq 3$ ) have been proved. In the context of Lotka-Volterra competition models, the effect of a chemorepulsive signal has been considered by Tello and Wrzosek in [31], proving the existence of global classical solution for the model in $d D$ domains (for $d \geq 1$ ). A chemorepulsion model with nonlinear chemotactic sensitivity has been studied in [30], obtaining the existence of bounded classical solution and the convergence at infinite time to a constant steady state in $d D$ domains (for $d \geq 3$ ).

With respect to the study of chemotaxis models with nonlinear signal production $\left(u^{p}\right)$ the literature is scarce (we refer [23, 32]). In [32], Winkler studied radially symmetric solutions of a parabolic-elliptic system, proving the existence of global bounded classical solution under some conditions on the power $p$. Considering nonlinear chemotactic sensitivity, chemorepulsion and nonlinear production, in [23] Lai and Xiao analyzed the existence, uniform boundedness and asymptotic behavior of 
global classical solutions also for a parabolic-elliptic model. However, as far as we know, there are not works studying the parabolic-parabolic problem (3) with production $u^{p}$ (for $1<p<2$ ). Therefore, the first aim of this work is to study the existence of global weak solutions of (3) (in the three-dimensional case) and global regularity (in the two and one-dimensional cases).

On the other hand, the second aim is to design numerical methods for model (3) conserving properties of the continuous problem such as: mass-conservation, energystability and positivity. It is important to mention that approaching chemorepulsion problems by using Finite Element (FE) approximations is not an easy task, because negative (discrete) solutions can be computed (see [17, 19, 20]). In those cases, some spurious oscillations may appear (see, for instance, [19] for a chemorepulsion model with quadratic production).

Some numerical schemes have been studied for chemotaxis models. Existence of discrete solutions, convergence, mass-conservation and error estimates, among other qualitative properties, have been studied in the context of Finite Volume (FV) schemes [13, 22, 34], Finite Element (FE) approximations [11, 25, 27, 28, 33] or combined FV-FE schemes [7].

Energy-stable numerical approximations have also been studied in the chemotaxis context. A conditionally energy-stable FV scheme for a chemo-attraction model with an additional cross-diffusion term was analyzed by Bessemoulin and Jüngel [6]. Energy-stability of time-discrete numerical approximations and fully discrete FE schemes for a chemorepulsion model with quadratic production have been analyzed in [17] and [18, 19], respectively; while, in the case of linear production, we refer [20]. However, as far as we know, for the chemorepulsion model with production term $u^{p}$ given in (3) there are not works studying energy-stable numerical schemes.

Likewise, the positivity or approximated positivity properties have been studied on numerical schemes for chemotaxis models. In [8], Chamoun and collaborators proved a discrete maximum principle for a combined FV-FE scheme approaching a chemotaxis-fluid model. The positivity of only time-discrete schemes and approximated positivity of a fully discrete FE scheme associated with a chemorepulsion model with quadratic signal production were proved in [17] and [19], respectively; while, for the case of linear production, we refer to [20]. Positive numerical methods, using FE techniques, associated with a generalized Keller-Segel model were studied in [10]. In [34], a positive FV scheme for a parabolic-elliptic chemotaxis model was analyzed. However, there are not works studying positive (or approximately positive) FE schemes for model (3).

The idea here is to extend the analysis made in [20], although in this case, we need to use two matrix operators (see (51) and (52) below) in order to obtain energystability and approximated positivity. The first one is the operator defined in [2] (and used in [20]); while, the second one, is obtained by constructing regularized functions associated with the test function $u^{p-1}$. For the second operator, it was necessary to prove technical Lemmas (see Lemmas 4.4 and 4.10 below) which are requiered in order to obtain the desired properties for the numerical schemes. 
Consequently, the main novelties in this paper are the following:

- The analysis of the existence of weak solutions of model (3) in the 3D case (which are regular and unique in the 2D and 1D cases) satisfying, in particular, an energy inequality (see (8) below).

- The introduction of a FE scheme (see scheme UVE in Section 4.1 below) for model (3) which is energy-stable with respect to an energy in the primitive variables $(u, v)$ and approximately positive, under a right-angled constraint on the spatial triangulation (see hypothesis $(\mathbf{H})$ in (46) below).

- The introduction of another FE scheme (see scheme $\mathbf{U S} \varepsilon$ in Section 4.2 below) for model (3) which is unconditionally energy-stable with respect to a modified energy and approximately positive, without imposing the restriction $(\mathbf{H})$ on the mesh.

The outline of this paper is as follows: In Section 2, we give the notation and some preliminary results. In Section 3, we prove the existence of weak-strong solutions of model (3) (in the sense of Definition 3.1 below) by using a regularization technique. In Section 4, we propose two fully discrete FE nonlinear approximations of problem (3), where the first one is defined in the variables $(u, v)$, and the second one introduces $\sigma=\nabla v$ as an auxiliary variable. We prove some unconditional properties such as mass-conservation, energy-stability, approximated positivity and solvability of the schemes. In Section 5, we compare the behavior of the schemes with respect to classical FE backward Euler scheme throughout several numerical simulations, including experimental convergence rates; and in Section 6, the main conclusions are summarized.

\section{Notation and preliminary results}

Along this paper, we will consider the usual Lebesgue spaces $L^{q}(\Omega), 1 \leq q \leq \infty$, with norm $\|\cdot\|_{L^{q}}$. In particular, the $L^{2}(\Omega)$-norm will be denoted by $\|\cdot\|_{0}$. From now on, $(\cdot, \cdot)$ will denote the standard $L^{2}$-inner product over $\Omega$. The usual Sobolev spaces $W^{m, p}(\Omega)=\left\{u \in L^{p}(\Omega):\left\|\partial^{\alpha} u\right\|_{L^{p}}<+\infty, \forall|\alpha| \leq m\right\}$, for a multi-index $\alpha, m \in \mathbb{N}$ and $p \geq 1$, with norm denoted by $\|\cdot\|_{W^{m, p}}$ will be also considered. If $m \geq 0$ is not integer, the space $W^{m, p}(\Omega)$ is a subspace of $W^{[m], p}(\Omega)$ (where $[m]$ is the integer part of $m$ ) of functions with finite norm (see [26]):

$$
\|u\|_{W^{m, p}}:=\left(\|u\|_{W^{[m], p}}^{p}+\sum_{|\alpha|=[m]} \int_{\Omega} \int_{\Omega} \frac{\left|D^{\alpha} u(x)-D^{\alpha} u(y)\right|^{p}}{|x-y|^{d+p(m-[m])}} d x d y\right)^{1 / p} .
$$

In the case when $p=2$, we denote $H^{m}(\Omega):=W^{m, 2}(\Omega)$, with respective norm $\|\cdot\|_{m}$. Moreover, the following spaces are set

$$
\begin{aligned}
W_{\mathbf{n}}^{m, p}(\Omega):= & \left.\left\{u \in W^{m, p}(\Omega): \frac{\partial u}{\partial \mathbf{n}}=0 \text { on } \partial \Omega\right\} \text { (for } m>1+1 / p\right), \\
& \boldsymbol{H}_{\sigma}^{1}(\Omega):=\left\{\boldsymbol{\sigma} \in \boldsymbol{H}^{1}(\Omega): \boldsymbol{\sigma} \cdot \mathbf{n}=0 \text { on } \partial \Omega\right\},
\end{aligned}
$$


and the following equivalent norms in $H^{1}(\Omega)$ and $\boldsymbol{H}_{\sigma}^{1}(\Omega)$, respectively, will be used (see [26] and [1, Corollary 3.5], respectively):

$$
\begin{gathered}
\|u\|_{1}^{2}=\|\nabla u\|_{0}^{2}+\left(\int_{\Omega} u\right)^{2}, \quad \forall u \in H^{1}(\Omega), \\
\|\boldsymbol{\sigma}\|_{1}^{2}=\|\boldsymbol{\sigma}\|_{0}^{2}+\|\operatorname{rot} \boldsymbol{\sigma}\|_{0}^{2}+\|\nabla \cdot \boldsymbol{\sigma}\|_{0}^{2}, \quad \forall \boldsymbol{\sigma} \in \boldsymbol{H}_{\sigma}^{1}(\Omega) .
\end{gathered}
$$

Here rot $\sigma$ denotes the well-known rotational operator (also called curl) which is a scalar operator for 2D domains and vectorial for 3D ones. In particular, (4) implies that, for all $\boldsymbol{\sigma}=\nabla v \in \boldsymbol{H}_{\sigma}^{1}(\Omega)$,

$$
\|\nabla v\|_{1}^{2}=\|\nabla v\|_{0}^{2}+\|\Delta v\|_{0}^{2}
$$

If $Z$ is a general Banach space, its topological dual space will be denoted by $Z^{\prime}$. Moreover, the letters $C, K$ will denote different positive constants which may change from line to line. The following result will be used along this paper:

Theorem 2.1 [12] Let $1<q<+\infty(q \neq 3)$ and suppose that $f \in$ $L^{q}\left(0, T ; L^{q}(\Omega)\right), u_{0} \in \widehat{W}^{2-\frac{2}{q}, q}(\Omega)$, where

$$
\widehat{W}^{2-\frac{2}{q}, q}(\Omega):=\left\{\begin{array}{lll}
W^{2-\frac{2}{q}, q}(\Omega) & \text { if } & q<3 \\
W_{\mathbf{n}}^{2-\frac{2}{q}, q}(\Omega) & \text { if } & q>3
\end{array}\right.
$$

Then, the problem

$$
\left\{\begin{array}{l}
\partial_{t} u-\Delta u=f \quad \text { in } \Omega, t>0 \\
\frac{\partial u}{\partial \mathbf{n}}=0 \text { on } \partial \Omega, t>0 \\
u(\boldsymbol{x}, 0)=u_{0}(\boldsymbol{x}) \text { in } \Omega
\end{array}\right.
$$

admits a unique solution $u$ in the class

$$
u \in L^{q}\left(0, T ; W^{2, q}(\Omega)\right) \cap C\left([0, T] ; \widehat{W}^{2-\frac{2}{q}, q}(\Omega)\right), \quad \partial_{t} u \in L^{q}\left(0, T ; L^{q}(\Omega)\right) .
$$

Moreover, there exists a positive constant $C=C(q, \Omega, T)$ such that

$$
\begin{aligned}
& \|u\|_{C\left([0, T] ; \widehat{W}^{2-\frac{2}{q}, q}(\Omega)\right)}+\left\|\partial_{t} u\right\|_{L^{q}\left(0, T ; L^{q}(\Omega)\right)}+\|u\|_{L^{q}\left(0, T ; W^{2, q}(\Omega)\right)} \\
\leq & C\left(\|f\|_{L^{q}\left(0, T ; L^{q}(\Omega)\right)}+\left\|u_{0}\right\|_{\widehat{W}^{2-\frac{2}{q}, q}(\Omega)}\right) .
\end{aligned}
$$

When large time estimates will be treated, the following result will be used (see [21]):

Lemma 2.2 Assume that $\delta, \beta, k>0$ and $d^{n} \geq 0$ satisfy

$$
(1+\delta k) d^{n+1} \leq d^{n}+k \beta, \quad \forall n \geq 0 .
$$

Then, for any $n_{0} \geq 0$,

$$
d^{n} \leq(1+\delta k)^{-\left(n-n_{0}\right)} d^{n_{0}}+\delta^{-1} \beta, \quad \forall n \geq n_{0} .
$$




\section{Analysis of the continuous model}

In this section, the existence of weak-strong solutions of problem (3) will be proved in the sense of the following definition.

Definition 3.1 (Weak-strong solutions of (3)) Let $1<p<2$. Given $\left(u_{0}, v_{0}\right) \in$ $L^{p}(\Omega) \times H^{1}(\Omega)$ with $u_{0} \geq 0, v_{0} \geq 0$ a.e. in $\Omega$,

a pair $(u, v)$ is called weak-strong solution of problem (3) in $(0,+\infty)$, if $u \geq 0$, $v \geq 0$ a.e. in $(0,+\infty) \times \Omega$,

$$
\begin{gathered}
u \in L^{\infty}\left(0,+\infty ; L^{p}(\Omega)\right) \cap L^{\frac{5 p}{p+3}}\left(0, T ; W^{1, \frac{5 p}{p+3}}(\Omega)\right), \quad \forall T>0, \\
v \in L^{\infty}\left(0,+\infty ; H^{1}(\Omega)\right) \cap L^{2}\left(0, T ; H^{2}(\Omega)\right), \quad \forall T>0, \\
\partial_{t} u \in L^{\frac{10 p}{3 p+6}}\left(0, T ; W^{1, \frac{10 p}{7 p-6}}(\Omega)^{\prime}\right), \partial_{t} v \in L^{\frac{5}{3}}\left(0, T ; L^{\frac{5}{3}}(\Omega)\right), \quad \forall T>0,
\end{gathered}
$$

the following variational formulation for the $u$-equation holds

$$
\begin{aligned}
\int_{0}^{T}\left\langle\partial_{t} u, \bar{u}\right\rangle & +\int_{0}^{T}(\nabla u, \nabla \bar{u}) \\
& +\int_{0}^{T}(u \nabla v, \nabla \bar{u})=0, \forall \bar{u} \in L^{\frac{10 p}{7 p-6}}\left(0, T ; W^{1, \frac{10 p}{7 p-6}}(\Omega)\right), \forall T>0,(6)
\end{aligned}
$$

the $v$-equation holds pointwisely

$$
\partial_{t} v-\Delta v+v=u^{p} \quad \text { a.e. }(t, \boldsymbol{x}) \in(0,+\infty) \times \Omega,
$$

the boundary condition $\frac{\partial v}{\partial \mathbf{n}}=0$ and the initial conditions (3) 4 are satisfied, and the following energy inequality (in integral version) holds a.e. $t_{0}, t_{1}$ with $t_{1} \geq t_{0} \geq 0$ :

$$
\mathcal{E}\left(u\left(t_{1}\right), v\left(t_{1}\right)\right)-\mathcal{E}\left(u\left(t_{0}\right), v\left(t_{0}\right)\right)+\int_{t_{0}}^{t_{1}}\left(\frac{4}{p}\left\|\nabla\left(u^{p / 2}(s)\right)\right\|_{0}^{2}+\|\nabla v(s)\|_{1}^{2}\right) d s \leq 0,
$$

where

$$
\mathcal{E}(u, v)=\frac{1}{p-1}\|u\|_{p}^{p}+\frac{1}{2}\|\nabla v\|_{0}^{2}
$$

Observe that any weak-strong solution of (3) is conservative in $u$, because the total mass $\int_{\Omega} u(\cdot, t)$ remains constant in time. In fact, by taking $\bar{u}=1$ in (6):

$$
\frac{d}{d t}\left(\int_{\Omega} u(\cdot, t)\right)=0, \quad \text { i.e., } \int_{\Omega} u(\cdot, t)=\int_{\Omega} u_{0}:=m_{0}, \quad \forall t>0 .
$$

In addition, integrating (7) in $\Omega$, one has

$$
\frac{d}{d t}\left(\int_{\Omega} v\right)+\int_{\Omega} v=\int_{\Omega} u^{p}
$$




\subsection{Regularized problem}

In order to prove the existence of weak-strong solution of problem (3) in the sense of Definition 3.1, we introduce the following regularized problem associated with model (3): Let $\varepsilon \in(0,1)$, find $\left(u^{\varepsilon}, z^{\varepsilon}\right)$, with $u^{\varepsilon} \geq 0$ a.e. in $(0,+\infty) \times \Omega$, such that, for all $T>0$,

$$
u^{\varepsilon}, z^{\varepsilon} \in \tilde{\mathcal{X}}:=\left\{w \in L^{\infty}\left(0, T ; W^{\frac{4}{5}, \frac{5}{3}}(\Omega)\right) \cap L^{\frac{5}{3}}\left(0, T ; W^{2, \frac{5}{3}}(\Omega)\right): \partial_{t} w \in L^{\frac{5}{3}}\left(0, T ; L^{\frac{5}{3}}(\Omega)\right)\right\},
$$

and satisfying the system

$$
\left\{\begin{array}{l}
\partial_{t} u^{\varepsilon}-\Delta u^{\varepsilon}=\nabla \cdot\left(u^{\varepsilon} \nabla v\left(z^{\varepsilon}\right)\right) \quad \text { in } \Omega, t>0 \\
\partial_{t} z^{\varepsilon}-\Delta z^{\varepsilon}+z^{\varepsilon}=\left(u^{\varepsilon}\right)^{p} \quad \text { in } \Omega, t>0 \\
\frac{\partial u^{\varepsilon}}{\partial \mathbf{n}}=\frac{\partial z^{\varepsilon}}{\partial \mathbf{n}}=0 \quad \text { on } \partial \Omega, t>0 \\
u^{\varepsilon}(\boldsymbol{x}, 0) \stackrel{=}{=} u_{0}^{\varepsilon}(\boldsymbol{x}) \geq 0, z^{\varepsilon}(\boldsymbol{x}, 0)=v_{0}^{\varepsilon}(\boldsymbol{x})-\varepsilon \Delta v_{0}^{\varepsilon}(\boldsymbol{x}) \quad \text { in } \Omega
\end{array}\right.
$$

where $v^{\varepsilon}=v\left(z^{\varepsilon}\right)$ is the unique solution of the elliptic-Newman problem

$$
\left\{\begin{array}{l}
v^{\varepsilon}-\varepsilon \Delta v^{\varepsilon}=z^{\varepsilon} \text { in } \Omega \\
\frac{\partial v^{\varepsilon}}{\partial \mathbf{n}}=0 \text { on } \partial \Omega
\end{array}\right.
$$

and $\left(u_{0}^{\varepsilon}, z_{0}^{\varepsilon}\right) \in W^{\frac{4}{5}, \frac{5}{3}}(\Omega)^{2}$ with

$$
\left(u_{0}^{\varepsilon}, z_{0}^{\varepsilon}\right) \rightarrow\left(u_{0}, v_{0}\right) \text { in } L^{p}(\Omega) \times H^{1}(\Omega)^{\prime}, \text { as } \varepsilon \rightarrow 0 .
$$

Notice that from (12), system (13) is satisfied a.e. in $(0,+\infty) \times \Omega$. From now on in this section, we will denote $v^{\varepsilon}\left(z^{\varepsilon}\right)$ solution of (14) only by $v^{\varepsilon}$. Observe that if $\left(u^{\varepsilon}, z^{\varepsilon}\right)$ is any solution of (13), then (10) and (11) are satisfied for $(u, v)=\left(u^{\varepsilon}, v^{\varepsilon}\right)$.

Theorem 3.2 There exists at least one solution of problem (12) and (13).

Proof We will use the Leray-Schauder fixed point theorem. With this aim, we denote

$$
\mathcal{X}:=L^{\infty}\left(0, T ; L^{2}(\Omega)\right) \cap L^{2}\left(0, T ; H^{1}(\Omega)\right),
$$

and we define the operator $R: \mathcal{X} \times \mathcal{X} \rightarrow \tilde{\mathcal{X}} \times \tilde{\mathcal{X}} \hookrightarrow \mathcal{X} \times \mathcal{X}$ by $R\left(\widetilde{u}^{\varepsilon}, \widetilde{z}^{\varepsilon}\right)=\left(u^{\varepsilon}, z^{\varepsilon}\right)$, such that $\left(u^{\varepsilon}, z^{\varepsilon}\right)$ solves the following linear decoupled problem

$$
\left\{\begin{array}{l}
\partial_{t} u^{\varepsilon}-\Delta u^{\varepsilon}=\nabla \cdot\left(\widetilde{u}_{+}^{\varepsilon} \nabla \widetilde{v}^{\varepsilon}\right) \text { in } \Omega, t>0, \\
\partial_{t} z^{\varepsilon}-\Delta z^{\varepsilon}=\left(\widetilde{u}_{+}^{\varepsilon}\right)^{p}-\widetilde{z}^{\varepsilon} \text { in } \Omega, t>0, \\
\frac{\partial u^{\varepsilon}}{\partial \mathbf{n}}=\frac{\partial z^{\varepsilon}}{\partial \mathbf{n}}=0 \text { on } \partial \Omega, t>0, \\
u^{\varepsilon}(\boldsymbol{x}, 0)=u_{0}^{\varepsilon}(\boldsymbol{x}) \geq 0, z^{\varepsilon}(\boldsymbol{x}, 0)=v_{0}^{\varepsilon}(\boldsymbol{x})-\varepsilon \Delta v_{0}^{\varepsilon}(\boldsymbol{x}) \text { in } \Omega,
\end{array}\right.
$$


where $\widetilde{v}^{\varepsilon}=v\left(\widetilde{z}^{\varepsilon}\right)$ and, in general, we denote $a_{+}:=\max \{a, 0\}$. Then, $\left(u^{\varepsilon}, z^{\varepsilon}\right)$ is a solution of (13) iff $\left(u^{\varepsilon}, z^{\varepsilon}\right)$ is a fixed point of the operator $R$ defined in (16). Let us check every hypotheses of Leray-Schauder Theorem:

1. $R$ is well defined. Observe that if $\widetilde{z}^{\varepsilon} \in \mathcal{X}$, from the $H^{2}$ and $H^{3}$-regularity of problem (14) (see [15, Theorems 2.4.2.7 and 2.5.1.1] respectively), we have that

$$
\widetilde{v}^{\varepsilon} \in L^{\infty}\left(0, T ; H^{2}(\Omega)\right) \cap L^{2}\left(0, T ; H^{3}(\Omega)\right) .
$$

Thus, we deduce that $\nabla \widetilde{v}^{\varepsilon} \in L^{\infty}\left(0, T ; H^{1}(\Omega)\right) \cap L^{2}\left(0, T ; H^{2}(\Omega)\right) \hookrightarrow$ $L^{10}\left(0, T ; L^{10}(\Omega)\right)$. Then, using this fact and taking into account that $\left(\widetilde{u}^{\varepsilon}, \widetilde{z}^{\varepsilon}\right) \in$ $\mathcal{X} \times \mathcal{X} \hookrightarrow L^{10 / 3}\left(0, T ; L^{10 / 3}(\Omega)\right)^{2}$, we obtain that $\nabla \cdot\left(\tilde{u}_{+}^{\varepsilon} \nabla \widetilde{v}^{\varepsilon}\right)=\nabla \widetilde{u}_{+}^{\varepsilon} \nabla \widetilde{v}^{\varepsilon}+$ $\tilde{u}_{+}^{\varepsilon} \Delta \widetilde{v}^{\varepsilon} \in L^{\frac{5}{3}}\left(0, T ; L^{\frac{5}{3}}(\Omega)\right)$ and $\left(\tilde{u}^{\varepsilon}\right)^{p}+\widetilde{z}^{\varepsilon} \in L^{\frac{5}{3}}\left(0, T ; L^{\frac{5}{3}}(\Omega)\right)$ for any $p \in(1,2)$ (using that $\widetilde{u}_{+}^{\varepsilon}, \Delta \widetilde{v}^{\varepsilon} \in L^{\frac{10}{3}}\left(0, T ; L^{\frac{10}{3}}(\Omega)\right)$ ). Thus, applying Theorem 2.1 to (16), we deduce that there exists a unique solution $\left(u^{\varepsilon}, z^{\varepsilon}\right)$ of (16), $\left(u^{\varepsilon}, z^{\varepsilon}\right) \in \widetilde{\mathcal{X}} \times \widetilde{\mathcal{X}}$ (where $\widetilde{\mathcal{X}}$ is defined in (12)).

2. All possible fixed points of $\lambda R$ (with $\lambda \in(0,1]$ ) are bounded in $\mathcal{X} \times \mathcal{X}$ and $u^{\varepsilon} \geq 0$. In fact, observe that if $\left(u^{\varepsilon}, z^{\varepsilon}\right)$ is a fixed point of $\lambda R$, then $\left(u^{\varepsilon}, z^{\varepsilon}\right)$ satisfies

$$
\left\{\begin{array}{l}
\partial_{t} u^{\varepsilon}-\Delta u^{\varepsilon}=\lambda \nabla \cdot\left(u_{+}^{\varepsilon} \nabla v^{\varepsilon}\right) \text { in } \Omega, t>0, \\
\partial_{t} z^{\varepsilon}-\Delta z^{\varepsilon}=\lambda\left(u_{+}^{\varepsilon}\right)^{p}-\lambda z^{\varepsilon} \text { in } \Omega, t>0, \\
\frac{\partial u^{\varepsilon}}{\partial \mathbf{n}}=\frac{\partial z^{\varepsilon}}{\partial \mathbf{n}}=0 \text { on } \partial \Omega, t>0, \\
u^{\varepsilon}(\boldsymbol{x}, 0)=u_{0}^{\varepsilon}(\boldsymbol{x}) \geq 0, z^{\varepsilon}(\boldsymbol{x}, 0)=v_{0}^{\varepsilon}(\boldsymbol{x})-\varepsilon \Delta v_{0}^{\varepsilon}(\boldsymbol{x}) \text { in } \Omega,
\end{array}\right.
$$

Multiplying $(17)_{1}$ by $u_{-}^{\varepsilon}:=\min \left\{u^{\varepsilon}, 0\right\}$ and integrating in $\Omega$, we have

$$
\frac{1}{2} \frac{d}{d t}\left\|u_{-}^{\varepsilon}\right\|_{0}^{2}+\left\|\nabla u_{-}^{\varepsilon}\right\|_{0}^{2}=\lambda\left(u_{+}^{\varepsilon} \nabla v^{\varepsilon}, \nabla u_{-}^{\varepsilon}\right)=0,
$$

which, taking into account that $u_{0}^{\varepsilon}(\boldsymbol{x}) \geq 0$ a.e. in $\Omega$, implies that $u^{\varepsilon} \geq$ 0 a.e. in $(0,+\infty) \times \Omega$. Thus, $u_{+}^{\varepsilon}=u^{\varepsilon}$. Now, we test (17) 1 and $(17)_{2}$ by $\frac{p}{p-1}\left(u^{\varepsilon}\right)^{p-1}$ and $-\Delta v^{\varepsilon}$ respectively, and adding both equations, the terms $-\lambda \frac{p}{p-1}\left(u^{\varepsilon} \nabla v^{\varepsilon}, \nabla\left(u^{\varepsilon}\right)^{p-1}\right)$ and $\lambda\left(\nabla\left(u^{\varepsilon}\right)^{p}, \nabla v^{\varepsilon}\right)$ cancel, and taking into account (14), we obtain

$$
\begin{aligned}
& \frac{d}{d t} \mathcal{E}_{\varepsilon}\left(u^{\varepsilon}, v^{\varepsilon}\right)+\frac{4}{p} \int_{\Omega}\left|\nabla\left(\left(u^{\varepsilon}\right)^{p / 2}\right)\right|^{2} \\
& \quad+\varepsilon\left\|\nabla\left(\Delta v^{\varepsilon}\right)\right\|_{0}^{2}+\left\|\Delta v^{\varepsilon}\right\|_{0}^{2}+\lambda\left\|\nabla v^{\varepsilon}\right\|_{0}^{2}+\lambda \varepsilon\left\|\Delta v^{\varepsilon}\right\|_{0}^{2}=0
\end{aligned}
$$

where

$$
\mathcal{E}_{\varepsilon}\left(u^{\varepsilon}, v^{\varepsilon}\right):=\frac{1}{p-1}\left\|u^{\varepsilon}\right\|_{L^{p}}^{p}+\frac{1}{2}\left\|\nabla v^{\varepsilon}\right\|_{0}^{2}+\frac{\varepsilon}{2}\left\|\Delta v^{\varepsilon}\right\|_{0}^{2} .
$$

Moreover, we observe that the function $y^{\varepsilon}(t)=\left(\int_{\Omega} v^{\varepsilon}(\boldsymbol{x}, t) d \boldsymbol{x}\right)^{2}$ satisfies the time differential inequality

$$
\left(y^{\varepsilon}\right)^{\prime}(t)+y^{\varepsilon}(t) \leq w^{\varepsilon}(t),
$$


with $w^{\varepsilon}(t)=\left\|u^{\varepsilon}(t)\right\|_{L^{p}}^{2 p}$. In fact, it follows by multiplying (11) (for $(u, v)=$ $\left.\left(u^{\varepsilon}, v^{\varepsilon}\right)\right)$ by $\int_{\Omega} v^{\varepsilon}(\boldsymbol{x}, t) d \boldsymbol{x}$ and using the Young inequality. Therefore, $y^{\varepsilon}(t)=$ $y^{\varepsilon}(0) e^{-t}+\int_{0}^{t} e^{-(t-s)} w^{\varepsilon}(s) d s$, which implies that

$$
\left(\int_{\Omega} v^{\varepsilon}(\boldsymbol{x}, t) d \boldsymbol{x}\right)^{2} \leq\left(\int_{\Omega} v_{0}^{\varepsilon}(\boldsymbol{x}) d \boldsymbol{x}\right)^{2}+\left\|u^{\varepsilon}\right\|_{L^{\infty}\left(0,+\infty ; L^{p}\right)}^{2 p}, \quad \forall t \geq 0 .
$$

Then, from (18) and (19) and using (5), we deduce the following estimates with respect to $\lambda$ :

$$
\left\{\begin{array}{l}
\left(u^{\varepsilon}, v^{\varepsilon}\right) \text { is bounded in } L^{\infty}\left(0,+\infty ; L^{p}(\Omega) \times \boldsymbol{H}^{2}(\Omega)\right), \\
\left(u^{\varepsilon}\right)^{\frac{p}{2}} \text { is bounded in } L^{\infty}\left(0,+\infty ; L^{2}(\Omega)\right) \cap L^{2}\left(0, T ; H^{1}(\Omega)\right) \hookrightarrow L^{\frac{10}{3}}\left(0, T ; L^{\frac{10}{3}}(\Omega)\right), \\
u^{\varepsilon} \text { is bounded in } L^{p}\left(0, T ; L^{3 p}(\Omega)\right) \text { and } v^{\varepsilon} \text { is bounded in } L^{2}\left(0, T ; \boldsymbol{H}^{3}(\Omega)\right) .
\end{array}\right.
$$

Then, from (20) we conclude that $z^{\varepsilon}$ is bounded in $\mathcal{X}$. Moreover, testing (17) 1 by $u^{\varepsilon}$, we have

$$
\begin{aligned}
\frac{1}{2} \frac{d}{d t}\left\|u^{\varepsilon}\right\|_{0}^{2}+\left\|u^{\varepsilon}\right\|_{1}^{2}= & -\lambda\left(u^{\varepsilon} \nabla v^{\varepsilon}, \nabla u^{\varepsilon}\right)+\left\|u^{\varepsilon}\right\|_{0}^{2} \leq \frac{1}{2}\left\|u^{\varepsilon}\right\|_{1}^{2} \\
& +C\left(\left\|\nabla v^{\varepsilon}\right\|_{1}^{4}+1\right)\left\|u^{\varepsilon}\right\|_{0}^{2},
\end{aligned}
$$

from which, taking into account (20) and using the Gronwall Lemma, we deduce that $u^{\varepsilon}$ is bounded in $\mathcal{X}$.

3. $R$ is compact. Let $\left\{\left(\widetilde{u}_{n}^{\varepsilon}, \widetilde{z}_{n}^{\varepsilon}\right)\right\}_{n \in \mathbb{N}}$ be a bounded sequence in $\mathcal{X} \times \mathcal{X}$. Then $\left(u_{n}^{\varepsilon}, z_{n}^{\varepsilon}\right)=R\left(\widetilde{u}_{n}^{\varepsilon}, \widetilde{z}_{n}^{\varepsilon}\right)$ solves (16) (with $\left(\tilde{u}_{n}^{\varepsilon}, \widetilde{z}_{n}^{\varepsilon}\right)$ and $\left(u_{n}^{\varepsilon}, z_{n}^{\varepsilon}\right)$ instead of $\left(\tilde{u}^{\varepsilon}, \widetilde{z}^{\varepsilon}\right)$ and $\left(u^{\varepsilon}, z^{\varepsilon}\right)$ respectively). Therefore, analogously as in item 1, we obtain that $\nabla \cdot\left(\widetilde{u}_{n+}^{\varepsilon} \nabla \widetilde{v}_{n}^{\varepsilon}\right)$ and $\left(\widetilde{u}_{n}^{\varepsilon}\right)^{p}+\widetilde{z}_{n}^{\varepsilon}$ are bounded in $L^{\frac{5}{3}}\left(0, T ; L^{\frac{5}{3}}(\Omega)\right)$; and therefore, from Theorem 2.1 we conclude that $\left\{R\left(\widetilde{u}_{n}^{\varepsilon}, \widetilde{z}_{n}^{\varepsilon}\right)\right\}_{n \in \mathbb{N}}$ is bounded in $\widetilde{\mathcal{X}} \times \tilde{\mathcal{X}}$ which is compactly embedded in $\mathcal{X} \times \mathcal{X}$, and thus $R$ is compact. Observe that the compactness embedding comes from the continuous embedding (using embeddings $W^{k, p}(\Omega) \hookrightarrow H^{s}(\Omega)$, see [24, Theorem 9.6]):

$$
\tilde{\mathcal{X}} \hookrightarrow L^{\infty}\left(0, T ; H^{1 / 2}(\Omega)\right) \cap L^{5 / 3}\left(0, T ; H^{17 / 10}(\Omega)\right) \hookrightarrow L^{2}\left(0, T ; H^{3 / 2}(\Omega)\right) .
$$

Then $u^{\varepsilon}, z^{\varepsilon} \in L^{\infty}\left(0, T ; H^{1 / 2}(\Omega)\right) \cap L^{2}\left(0, T ; H^{3 / 2}(\Omega)\right)$ and $\partial_{t} u^{\varepsilon}, \partial_{t} z^{\varepsilon} \in$ $L^{5 / 3}\left(0, T ; L^{5 / 3}(\Omega)\right)$, hence the compactness holds by applying the Aubin-Lions Lemma (see [29]).

4. $R$ is continuous from $\mathcal{X} \times \mathcal{X}$ into $\mathcal{X} \times \mathcal{X}$. Let $\left\{\left(\widetilde{u}_{n}^{\varepsilon}, \widetilde{z}_{n}^{\varepsilon}\right)\right\}_{n \in \mathbb{N}} \subset \mathcal{X} \times \mathcal{X}$ be a sequence such that

$$
\left(\widetilde{u}_{n}^{\varepsilon}, \widetilde{z}_{n}^{\varepsilon}\right) \rightarrow\left(\widetilde{u}^{\varepsilon}, \widetilde{z}^{\varepsilon}\right) \text { in } \mathcal{X} \times \mathcal{X}, \quad \text { as } n \rightarrow+\infty .
$$

Therefore, $\left\{\left(\tilde{u}_{n}^{\varepsilon}, \widetilde{z}_{n}^{\varepsilon}\right)\right\}_{n \in \mathbb{N}}$ is bounded in $\mathcal{X} \underset{\sim}{\mathcal{X}}$, and from item 3 we deduce that $\left\{\left(u_{n}^{\varepsilon}, z_{n}^{\varepsilon}\right)=R\left(\widetilde{u}_{n}^{\varepsilon}, \widetilde{z}_{n}^{\varepsilon}\right)\right\}_{n \in \mathbb{N}}$ is bounded in $\widetilde{\mathcal{X}} \times \widetilde{\mathcal{X}}$. Then, there exist $\left(\hat{u}^{\varepsilon}, \hat{z}^{\varepsilon}\right)$ and a subsequence of $\left\{R\left(\widetilde{u}_{n}^{\varepsilon}, \widetilde{z}_{n}^{\varepsilon}\right)\right\}_{n \in \mathbb{N}}$ still denoted by $\left\{R\left(\widetilde{u}_{n}^{\varepsilon}, \widetilde{z}_{n}^{\varepsilon}\right)\right\}_{n \in \mathbb{N}}$ such that

$$
R\left(\widetilde{u}_{n}^{\varepsilon}, \widetilde{z}_{n}^{\varepsilon}\right) \rightarrow\left(\hat{u}^{\varepsilon}, \hat{z}^{\varepsilon}\right) \text { weakly in } \tilde{\mathcal{X}} \times \tilde{\mathcal{X}} \text { and strongly in } \mathcal{X} \times \mathcal{X}
$$


Then, from (21) and (22), a standard procedure allows us to pass to the limit, as $n$ goes to $+\infty$, in (16) (with $\left(\widetilde{u}_{n}^{\varepsilon}, \widetilde{z}_{n}^{\varepsilon}\right)$ and $\left(u_{n}^{\varepsilon}, z_{n}^{\varepsilon}\right)$ instead of $\left(\widetilde{u}^{\varepsilon}, \widetilde{z}^{\varepsilon}\right)$ and $\left(u^{\varepsilon}, z^{\varepsilon}\right)$ respectively), and we deduce that $R\left(\widetilde{u}^{\varepsilon}, \widetilde{z}^{\varepsilon}\right)=\left(\hat{u}^{\varepsilon}, \hat{z}^{\varepsilon}\right)$. Therefore, we have proved that any convergent subsequence of $\left\{R\left(\widetilde{u}_{n}^{\varepsilon}, \widetilde{z}_{n}^{\varepsilon}\right)\right\}_{n \in \mathbb{N}}$ converges to $R\left(\widetilde{u}^{\varepsilon}, \widetilde{z}^{\varepsilon}\right)$ strong in $\mathcal{X} \times \mathcal{X}$, and from uniqueness of $R\left(\widetilde{u}^{\varepsilon}, \widetilde{z}^{\varepsilon}\right)$, we conclude that the whole sequence $R\left(\widetilde{u}_{n}^{\varepsilon}, \widetilde{z}_{n}^{\varepsilon}\right) \rightarrow R\left(\widetilde{u}^{\varepsilon}, \widetilde{z}^{\varepsilon}\right)$ in $\mathcal{X} \times \mathcal{X}$. Thus, $R$ is continuous.

Therefore, the hypotheses of the Leray-Schauder fixed point theorem are satisfied and we conclude that the map $R\left(\widetilde{u}^{\varepsilon}, \widetilde{z}^{\varepsilon}\right)$ has a fixed point $\left(u^{\varepsilon}, z^{\varepsilon}\right)$, that is, $R\left(u^{\varepsilon}, z^{\varepsilon}\right)=$ $\left(u^{\varepsilon}, z^{\varepsilon}\right)$, which is a solution of problem (12) and (13).

\subsection{Existence of weak-strong solutions of (3)}

Theorem 3.3 There exists at least one $(u, v)$ weak-strong solution of problem (3).

Proof Observe that a variational problem associated with (13) is:

$$
\left\{\begin{array}{l}
\int_{0}^{T}\left\langle\partial_{t} u^{\varepsilon}, \bar{u}\right\rangle+\int_{0}^{T}\left(\nabla u^{\varepsilon}, \nabla \bar{u}\right)+\int_{0}^{T}\left(u^{\varepsilon} \nabla v^{\varepsilon}, \nabla \bar{u}\right)=0, \quad \forall \bar{u} \in L^{\frac{10 p}{7 p-6}}\left(0, T ; W^{1, \frac{10 p}{7 p-6}}(\Omega)\right) \\
\int_{0}^{T}\left\langle\partial_{t} z^{\varepsilon}, \bar{z}\right\rangle+\int_{0}^{T}\left(\nabla z^{\varepsilon}, \nabla \bar{z}\right)+\int_{0}^{T}\left(z^{\varepsilon}, \bar{z}\right)=\int_{0}^{T}\left(\left(u^{\varepsilon}\right)^{p}, \bar{z}\right), \quad \forall \bar{z} \in L^{\frac{5}{2}}\left(0, T ; H^{1}(\Omega)\right) .
\end{array}\right.
$$

Recall that $v^{\varepsilon}=v\left(z^{\varepsilon}\right)$ is the unique solution of problem (14). From (18) we have that $\left(u^{\varepsilon}, v^{\varepsilon}\right)$ satisfies the following energy equality:

$$
\frac{d}{d t} \mathcal{E}_{\varepsilon}\left(u^{\varepsilon}, v^{\varepsilon}\right)+\frac{4}{p}\left\|\nabla\left(\left(u^{\varepsilon}\right)^{p / 2}\right)\right\|_{0}^{2}+\varepsilon\left\|\Delta v^{\varepsilon}\right\|_{1}^{2}+\left\|\nabla v^{\varepsilon}\right\|_{1}^{2}=0 .
$$

Then, from (24) and using (19) we deduce the following estimates (independent of $\varepsilon$ )

$$
\left\{\begin{array}{l}
\left\{\left(u^{\varepsilon}\right)^{\frac{p}{2}}\right\} \text { is bounded in } L^{\infty}\left(0,+\infty ; L^{2}(\Omega)\right) \cap L^{2}\left(0, T ; H^{1}(\Omega)\right) \hookrightarrow L^{\frac{10}{3}}\left(0, T ; L^{\frac{10}{3}}(\Omega)\right), \\
\left\{v^{\varepsilon}\right\} \text { is bounded in } L^{\infty}\left(0,+\infty ; H^{1}(\Omega)\right) \cap L^{2}\left(0, T ; H^{2}(\Omega)\right), \\
\left\{\sqrt{\varepsilon} \Delta v^{\varepsilon}\right\} \text { is bounded in } L^{\infty}\left(0,+\infty ; L^{2}(\Omega)\right) \cap L^{2}\left(0, T ; H^{1}(\Omega)\right)
\end{array}\right.
$$

and therefore,

$$
\left\{\begin{array}{l}
\left\{u^{\varepsilon}\right\} \text { is bounded in } L^{\infty}\left(0,+\infty ; L^{p}(\Omega)\right) \cap L^{p}\left(0, T ; L^{3 p}(\Omega)\right) \hookrightarrow L^{\frac{5 p}{3}}\left(0, T ; L^{\frac{5 p}{3}}(\Omega)\right), \\
\left\{z^{\varepsilon}\right\} \text { is bounded in } L^{\infty}\left(0,+\infty ; L^{2}(\Omega)\right) \cap L^{2}\left(0, T ; H^{1}(\Omega)\right), \\
\left\{\partial_{t} u^{\varepsilon}\right\} \text { is bounded in }\left[L^{\frac{10 p}{7 p-6}}\left(0, T ; W^{1, \frac{10 p}{7 p-6}}(\Omega)\right)\right]^{\prime} \\
\left\{\partial_{t} z^{\varepsilon}\right\} \text { is bounded in }\left[L^{\frac{5}{2}}\left(0, T ; H^{1}(\Omega)\right)\right]^{\prime} .
\end{array}\right.
$$

Moreover, taking into account that from $(25)_{1}$ we have that $\nabla\left(\left(u^{\varepsilon}\right)^{p / 2}\right)$ is bounded in $L^{2}\left(0, T ; L^{2}(\Omega)\right)$ and from $(26)_{1} u^{1-\frac{p}{2}}$ is bounded in $L^{\frac{10 p}{6-3 p}}\left(0, T ; L^{\frac{10 p}{6-3 p}}(\Omega)\right)$, we 
conclude that $\nabla u^{\varepsilon}=\frac{2}{p} u^{1-\frac{p}{2}} \nabla\left(\left(u^{\varepsilon}\right)^{p / 2}\right)$ is bounded in $L^{\frac{5 p}{p+3}}\left(0, T ; L^{\frac{5 p}{p+3}}(\Omega)\right)$. Therefore, we deduce that

$$
\left\{u^{\varepsilon}\right\} \text { is bounded in } L^{\frac{5 p}{p+3}}\left(0, T ; W^{1, \frac{5 p}{p+3}}(\Omega)\right) .
$$

Notice that from (14) and $(25)_{3}$, we can deduce that

$$
\left\|z^{\varepsilon}-v^{\varepsilon}\right\|_{L^{\infty} L^{2} \cap L^{2} H^{1}} \leq \varepsilon\left\|\Delta v^{\varepsilon}\right\|_{L^{\infty} L^{2} \cap L^{2} H^{1}} \rightarrow 0 \text { as } \varepsilon \rightarrow 0 .
$$

Then, from (25)-(28), we deduce that there exists $(u, v)$, with

$$
\left\{\begin{array}{l}
u \in L^{\infty}\left(0,+\infty ; L^{p}(\Omega)\right) \cap L^{\frac{5 p}{3}}\left(0, T ; L^{\frac{5 p}{3}}(\Omega)\right) \cap L^{\frac{5 p}{p+3}}\left(0, T ; W^{1, \frac{5 p}{p+3}}(\Omega)\right), \\
v \in L^{\infty}\left(0,+\infty ; H^{1}(\Omega)\right) \cap L^{2}\left(0, T ; H^{2}(\Omega)\right),
\end{array}\right.
$$

such that for some subsequence of $\left\{u^{\varepsilon}, z^{\varepsilon}, v^{\varepsilon}\right\}$ still denoted by $\left\{u^{\varepsilon}, z^{\varepsilon}, v^{\varepsilon}\right\}$, the following weak convergences hold when $\varepsilon \rightarrow 0$,

$$
\left\{\begin{array}{l}
u^{\varepsilon} \rightarrow u \text { weakly in } L^{\frac{5 p}{3}}\left(0, T ; L^{\frac{5 p}{3}}(\Omega)\right) \cap L^{\frac{5 p}{p+3}}\left(0, T ; W^{1, \frac{5 p}{p+3}}(\Omega)\right), \\
v^{\varepsilon} \rightarrow v \text { weakly in } L^{2}\left(0, T ; H^{2}(\Omega)\right), \\
z^{\varepsilon} \rightarrow v \text { weakly in } L^{2}\left(0, T ; H^{1}(\Omega)\right), \\
\partial_{t} u^{\varepsilon} \rightarrow \partial_{t} u \text { weakly- } \star \text { in }\left[L^{\frac{10 p}{7 p-6}}\left(0, T ; W^{1, \frac{10 p}{7 p-6}}(\Omega)\right)\right]^{\prime} \\
\partial_{t} z^{\varepsilon} \rightarrow \partial_{t} v \text { weakly- } \star \text { in }\left[L^{\frac{5}{2}}\left(0, T ; H^{1}(\Omega)\right)\right]^{\prime}
\end{array}\right.
$$

On the other hand, taking into account (26) 3 and (27), the Aubin-Lions Lemma implies that

$$
\left\{u^{\varepsilon}\right\} \text { is relatively compact in } L^{\frac{5 p}{p+3}}\left(0, T ; L^{2}(\Omega)\right)
$$

(and also in $L^{r}\left(0, T ; L^{r}(\Omega)\right.$ ), for all $\left.r<\frac{5 p}{3}\right)$. In particular, since $u^{\varepsilon} \geq 0$ then $u \geq 0$ a.e. in $(0,+\infty) \times \Omega$. Moreover, since the embedding $L^{\infty}\left(0, T ; L^{2}(\Omega)\right) \cap$ $L^{2}\left(0, T ; H^{1}(\Omega)\right) \hookrightarrow L^{\frac{10}{3}}\left(0, T ; L^{\frac{10}{3}}(\Omega)\right)$ is continuous, from $(25)_{2}$ we deduce that

$$
\nabla v^{\varepsilon} \rightarrow \nabla v \text { weakly in } L^{\frac{10}{3}}\left(0, T ; L^{\frac{10}{3}}(\Omega)\right) .
$$

Thus, from (30) and (31) and using that $u^{\varepsilon} \nabla v^{\varepsilon}$ is bounded in $L^{\frac{10 p}{3 p+6}}$ $\left(0, T ; \boldsymbol{L}^{\frac{10 p}{3 p+6}}(\Omega)\right)$, we deduce that

$$
u^{\varepsilon} \nabla v^{\varepsilon} \rightarrow u \nabla v \text { weakly in } L^{\frac{10 p}{3 p+6}}\left(0, T ; L^{\frac{10 p}{3 p+6}}(\Omega)\right) .
$$

Moreover, since $u^{\varepsilon} \rightarrow u$ strongly in $L^{p}\left(0, T ; L^{p}(\Omega)\right)$, we have that

$$
\left(u^{\varepsilon}\right)^{p} \rightarrow u^{p} \text { strongly in } L^{1}\left(0, T ; L^{1}(\Omega)\right)
$$


Thus, taking to the limit when $\varepsilon \rightarrow 0$ in (23), and using (29), (32) and (33), we obtain that $(u, v)$ satisfies

$$
\begin{gathered}
\int_{0}^{T}\left\langle\partial_{t} u, \bar{u}\right\rangle+\int_{0}^{T}(\nabla u, \nabla \bar{u})+\int_{0}^{T}(u \nabla v, \nabla \bar{u})=0, \forall \bar{u} \in L^{\frac{10 p}{7 p-6}}\left(0, T ; W^{1 \frac{10 p}{7 p-6}}(\Omega)\right), \\
\int_{0}^{T}\left\langle\partial_{t} v, \bar{z}\right\rangle+\int_{0}^{T}(\nabla v, \nabla \bar{z})+\int_{0}^{T}(v, \bar{z})=\int_{0}^{T}\left(u^{p}, \bar{z}\right), \quad \forall \bar{z} \in L^{\frac{5}{2}}\left(0, T ; H^{1}(\Omega)\right),
\end{gathered}
$$

and therefore, integrating by parts in (35) and taking into account that $u^{p} \in$ $L^{\frac{5}{3}}\left(0, T ; L^{\frac{5}{3}}(\Omega)\right)$ and $v \in L^{2}\left(0, T ; H^{2}(\Omega)\right)$, we arrive at

$$
\partial_{t} v-\Delta v+v=u^{p} \text { in } L^{\frac{5}{3}}\left(0, T ; L^{\frac{5}{3}}(\Omega)\right),
$$

with $\frac{\partial v}{\partial \boldsymbol{n}}=0$ on $\partial \Omega$. Notice that the limit function $v$ is nonnegative. In fact, it follows by testing (36) by $v_{-}$and using that $v_{0} \geq 0$. Finally, we will prove that $(u, v)$ satisfies the energy inequality (8). Indeed, integrating (24) in time from $t_{0}$ to $t_{1}$, with $t_{1}>$ $t_{0} \geq 0$, and taking into account that

$$
\int_{t_{0}}^{t_{1}} \frac{d}{d t} \mathcal{E}_{\varepsilon}\left(u^{\varepsilon}, v^{\varepsilon}\right)=\mathcal{E}_{\varepsilon}\left(u^{\varepsilon}\left(t_{1}\right), v^{\varepsilon}\left(t_{1}\right)\right)-\mathcal{E}_{\varepsilon}\left(u^{\varepsilon}\left(t_{0}\right), v^{\varepsilon}\left(t_{0}\right)\right) \quad \forall t_{0}<t_{1},
$$

since $\mathcal{E}_{\varepsilon}\left(u^{\varepsilon}(t), v^{\varepsilon}(t)\right) \in W^{1,1}(0, T)$ for all $T>0$, is continuous in time, we deduce

$$
\begin{aligned}
& \mathcal{E}_{\varepsilon}\left(u^{\varepsilon}\left(t_{1}\right), v^{\varepsilon}\left(t_{1}\right)\right)-\mathcal{E}_{\varepsilon}\left(u^{\varepsilon}\left(t_{0}\right), v^{\varepsilon}\left(t_{0}\right)\right) \\
& +\int_{t_{0}}^{t_{1}}\left(\frac{4}{p}\left\|\nabla\left(\left(u^{\varepsilon}(t)\right)^{p / 2}\right)\right\|_{0}^{2}+\varepsilon\left\|\Delta v^{\varepsilon}(t)\right\|_{1}^{2}+\left\|\nabla v^{\varepsilon}(t)\right\|_{1}^{2}\right) d t=0, \quad \forall t_{0}<t_{1} .
\end{aligned}
$$

Now, we will prove that

$$
\mathcal{E}_{\varepsilon}\left(u^{\varepsilon}(t), v^{\varepsilon}(t)\right) \rightarrow \mathcal{E}(u(t), v(t)), \text { a.e. } t \in[0,+\infty) .
$$

Since $u^{\varepsilon}$ is relatively compact in $L^{p}\left(0, T ; L^{p}(\Omega)\right)$, we have

$$
u^{\varepsilon} \rightarrow u \text { strongly in } L^{p}\left(0, T ; L^{p}(\Omega)\right) .
$$

Moreover, for any $T>0$,

$$
\begin{aligned}
\| \mathcal{E}_{\varepsilon} & \left(u^{\varepsilon}(t), v^{\varepsilon}(t)\right)-\mathcal{E}(u(t), v(t)) \|_{L^{1}(0, T)}=\int_{0}^{T}\left|\mathcal{E}_{\varepsilon}\left(u^{\varepsilon}(t), v^{\varepsilon}(t)\right)-\mathcal{E}(u(t), v(t))\right| d t \\
\leq & \int_{0}^{T}\left|\frac{1}{p-1}\left(\left\|u^{\varepsilon}(t)\right\|_{L^{p}}^{p}-\|u(t)\|_{L^{p}}^{p}\right)+\frac{1}{2}\left(\left\|\nabla v^{\varepsilon}(t)\right\|_{0}^{2}-\|\nabla v(t)\|_{0}^{2}\right)+\frac{\varepsilon}{2}\left\|\Delta v^{\varepsilon}\right\|_{0}^{2}\right| d t \\
\leq & C \frac{p}{p-1}\left\|u^{\varepsilon}-u\right\|_{L^{p}\left(0, T ; L^{p}\right)}\left(\left\|u^{\varepsilon}\right\|_{L^{p}\left(0, T ; L^{p}\right)}+\|u\|_{L^{p}\left(0, T ; L^{p}\right)}\right)^{p-1} \\
& +\frac{1}{2}\left\|\nabla v^{\varepsilon}-\nabla v\right\|_{L^{2}\left(0, T ; L^{2}\right)}\left(\left\|\nabla v^{\varepsilon}\right\|_{L^{2}\left(0, T ; L^{2}\right)}+\|\nabla v\|_{L^{2}\left(0, T ; L^{2}\right)}\right)+\frac{\varepsilon}{2}\left\|\Delta v^{\varepsilon}\right\|_{L^{2}\left(0, T ; L^{2}\right)^{2}}^{2}
\end{aligned}
$$

Then, taking into account that $u^{\varepsilon} \rightarrow u$ strongly in $L^{p}\left(0, T ; L^{p}(\Omega)\right), \nabla v^{\varepsilon} \rightarrow \nabla v$ strongly in $L^{2}\left(0, T ; L^{2}(\Omega)\right)$ for any $T>0$, and $\Delta v^{\varepsilon}$ is bounded in $L^{2}\left(0, T ; L^{2}(\Omega)\right)$, from (40) we conclude that $\mathcal{E}_{\varepsilon}\left(u^{\varepsilon}(t), v^{\varepsilon}(t)\right) \rightarrow \mathcal{E}(u(t), v(t))$ strongly in $L^{1}(0, T)$ for all $T>0$, which implies in particular (38). Finally, observe that from (39) we have 
that $\left(u^{\varepsilon}\right)^{p / 2} \rightarrow u^{p / 2}$ strongly in $L^{2}\left(0, T ; L^{2}(\Omega)\right)$; and since $\nabla\left(\left(u^{\varepsilon}\right)^{p / 2}\right.$ is bounded in $L^{2}\left(0, T ; L^{2}(\Omega)\right)$ we deduce that

$$
\nabla\left(\left(u^{\varepsilon}\right)^{p / 2}\right) \rightarrow \nabla\left(u^{p / 2}\right) \text { weakly in } L^{2}\left(0, T ; L^{2}(\Omega)\right) .
$$

Then, by using weakly lower semicontinuity,

$$
\begin{aligned}
& \liminf _{\varepsilon \rightarrow 0} \int_{t_{0}}^{t_{1}}\left(\frac{4}{p}\left\|\nabla\left(\left(u^{\varepsilon}(t)\right)^{p / 2}\right)\right\|_{0}^{2}+\varepsilon\left\|\Delta v^{\varepsilon}(t)\right\|_{1}^{2}+\left\|\nabla v^{\varepsilon}(t)\right\|_{1}^{2}\right) d t \\
\geq & \int_{t_{0}}^{t_{1}}\left(\frac{4}{p}\left\|\nabla\left(u(t)^{p / 2}\right)\right\|_{0}^{2}+\|\nabla v(t)\|_{1}^{2}\right) d t \quad \forall t_{1} \geq t_{0} \geq 0 .
\end{aligned}
$$

On the other hand, owing to (38),

$$
\liminf _{\varepsilon \rightarrow 0}\left[\mathcal{E}_{\varepsilon}\left(u^{\varepsilon}\left(t_{1}\right), v^{\varepsilon}\left(t_{1}\right)\right)-\mathcal{E}_{\varepsilon}\left(u^{\varepsilon}\left(t_{0}\right), v^{\varepsilon}\left(t_{0}\right)\right)\right]=\mathcal{E}\left(u\left(t_{1}\right), v\left(t_{1}\right)\right)-\mathcal{E}\left(u\left(t_{0}\right), v\left(t_{0}\right)\right)
$$

a.e. $t_{1}, t_{0}: t_{1} \geq t_{0} \geq 0$. Thus, taking liminf as $\varepsilon \rightarrow 0$ in inequality (37), we deduce the energy inequality (8) for a.e. $t_{0}, t_{1}: t_{1} \geq t_{0} \geq 0$.

Remark 3.4 (Regularity in $1 D$ and $2 D$ domains) In this work, we have proved existence of global in time weak solutions for model (1). In [5], the existence and uniqueness of a local in time positive classical solution $(u, v)$ is proved whenever $u_{0} \in C^{0}(\bar{\Omega})$ and $v_{0} \in W^{1, q}(\Omega)$ for $q>d$ (for $d \geq 1$ the space dimension), which is global in time under the extensibility criteria

$$
\|u(\cdot, t)\|_{L^{\infty}(\Omega)}+\|v(\cdot, t)\|_{W^{1, q}(\Omega)} \leq C \quad \text { for all } t \in(0, T) .
$$

Moreover, it is also proved in [5] that condition (41) holds if there exist $M>0$ and $\gamma \geq 1$ with $\gamma>d p / 2$ such that

$$
\|u(\cdot, t)\|_{L^{\gamma}(\Omega)} \leq M \quad \text { for all } t \in(0, T) .
$$

Observe that, in $1 D, 2 D$ or $3 D$ domains, condition (42) reads as $\|u(\cdot, t)\|_{L^{\gamma}(\Omega)} \leq$ $M$ for all $t \in(0, T)$, for $\gamma \geq 1, \gamma>p$ and $\gamma>3 p / 2$, respectively. Since the weakstrong regularity of $(u, v)$ given in Definition 3.1 only guarantees the boundedness of $\|u(\cdot, t)\|_{L^{p}(\Omega)} \leq M$, therefore this regularity result can only be applied for $1 D$ domains. On the other hand, in [9] it is proved that in $2 D$ domains, assuming $\Delta v \in$ $L^{2}\left(0, T ; L^{2}(\Omega)\right)$ and reasoning over the equation $(1)_{1}$, then $u \in L^{\infty}\left(0, T ; L^{q}(\Omega)\right)$ for any $q<\infty$. Consequently, since the weak-strong regularity guarantees that $\Delta v \in$ $L^{2}\left(0, T ; L^{2}(\Omega)\right)$ and the $u$-equation in our model and in [9] is the same, then one also has the existence and uniqueness of global in time regular solutions for (1) in $2 D$ domains.

\section{Fully discrete numerical schemes}

In this section, two fully discrete numerical schemes associated with model (3) are proposed. Some unconditional properties such as mass-conservation, energystability, approximated positivity and solvability of the schemes are proved. 


\subsection{Scheme UV $\varepsilon$}

In this section, in order to construct an energy-stable fully discrete scheme for model (3), we are going to make a regularization procedure, in which we will adapt the ideas of [2] (see also [16]). With this aim, given $\varepsilon \in(0,1)$ we consider a function $F_{\varepsilon}: \mathbb{R} \rightarrow[0,+\infty)$, approximation of $f(s)=s^{p}$, such that $F_{\varepsilon} \in C^{2}(\mathbb{R})$ and

$$
F_{\varepsilon}^{\prime \prime}(s):= \begin{cases}\varepsilon^{p-2} & \text { if } s \leq \varepsilon, \\ s^{p-2} & \text { if } \varepsilon \leq s \leq \varepsilon^{-1}, \\ \varepsilon^{2-p} & \text { if } s \geq \varepsilon^{-1}\end{cases}
$$

Then, $F_{\varepsilon}$ is obtained by integrating in (43) and imposing the conditions $F_{\varepsilon}^{\prime}(s)=$ $\frac{s^{p-1}}{p-1}$ and $F_{\varepsilon}(s)=\frac{s^{p}}{p(p-1)}$ for all $\varepsilon \leq s \leq \varepsilon^{-1}$ (see Fig. 1); and

$$
a_{\varepsilon}(s):=(p-1) \frac{F_{\varepsilon}^{\prime}(s)}{F_{\varepsilon}^{\prime \prime}(s)}= \begin{cases}(p-1) s+(2-p) \varepsilon^{3-p} & \text { if } s \leq \varepsilon, \\ s & \text { if } \varepsilon \leq s \leq \varepsilon^{-1}, \\ (p-1) s+(2-p) \varepsilon^{p-3} & \text { if } s \geq \varepsilon^{-1}\end{cases}
$$

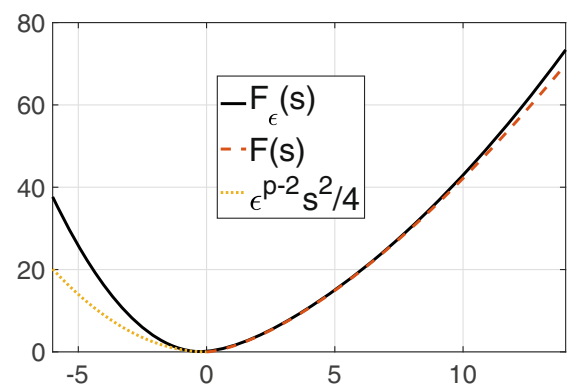

(a) $F_{\varepsilon}(s)$ vs $F(s):=\frac{1}{p(p-1)} s^{p}$

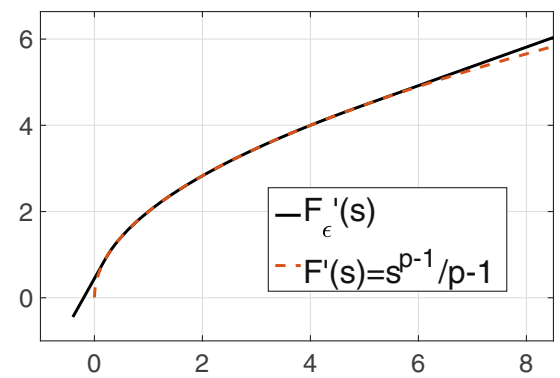

(b) $F_{\varepsilon}^{\prime}(s)$ vs $F^{\prime}(s):=\frac{1}{p-1} s^{p-1}$

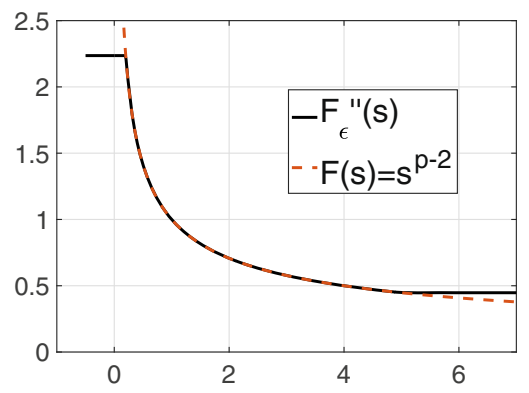

(c) $F_{\varepsilon}^{\prime \prime}(s)$ vs $F^{\prime \prime}(s):=s^{p-2}$

Fig. 1 The function $F_{\varepsilon}$ and its derivatives 
Then, taking into account the functions $F_{\varepsilon}$, its derivatives and $a_{\varepsilon}$, a regularized version of problem (3) reads: Find $u_{\varepsilon}: \Omega \times[0, T] \rightarrow \mathbb{R}$ and $v_{\varepsilon}: \Omega \times[0, T] \rightarrow \mathbb{R}$, with $u_{\varepsilon}, v_{\varepsilon} \geq 0$, such that

$$
\left\{\begin{array}{l}
\partial_{t} u_{\varepsilon}-\Delta u_{\varepsilon}-\nabla \cdot\left(a_{\varepsilon}\left(u_{\varepsilon}\right) \nabla v_{\varepsilon}\right)=0 \text { in } \Omega, t>0, \\
\partial_{t} v_{\varepsilon}-\Delta v_{\varepsilon}+v_{\varepsilon}=p(p-1) F_{\varepsilon}\left(u_{\varepsilon}\right) \text { in } \Omega, t>0, \\
\frac{\partial u_{\varepsilon}}{\partial \mathbf{n}}=\frac{\partial v_{\varepsilon}}{\partial \mathbf{n}}=0 \text { on } \partial \Omega, t>0, \\
u_{\varepsilon}(\boldsymbol{x}, 0)=u_{0}(\boldsymbol{x}) \geq 0, v_{\varepsilon}(\boldsymbol{x}, 0)=v_{0}(\boldsymbol{x}) \geq 0 \text { in } \Omega .
\end{array}\right.
$$

Remark 4.1 The idea is to define a fully discrete scheme associated with (45), taking in general $\varepsilon=\varepsilon(k, h)$, such that $\varepsilon(k, h) \rightarrow 0$ as $(k, h) \rightarrow 0$, where $k$ is the time step and $h$ the mesh size.

Observe that (at least formally) multiplying (45) 1 by $p F_{\varepsilon}^{\prime}\left(u_{\varepsilon}\right),(45)_{2}$ by $-\Delta v_{\varepsilon}$, integrating over $\Omega$ and adding, the chemotaxis and production terms cancel and we obtain the following energy law

$$
\frac{d}{d t} \int_{\Omega}\left(p F_{\varepsilon}\left(u_{\varepsilon}\right)+\frac{1}{2}\left|\nabla v_{\varepsilon}\right|^{2}\right) d \boldsymbol{x}+\int_{\Omega} p F_{\varepsilon}^{\prime \prime}\left(u_{\varepsilon}\right)\left|\nabla u_{\varepsilon}\right|^{2} d \boldsymbol{x}+\left\|\nabla v_{\varepsilon}\right\|_{1}^{2}=0
$$

In particular, the modified energy

$$
\mathcal{E}_{\varepsilon}(u, v)=\int_{\Omega}\left(p F_{\varepsilon}(u)+\frac{1}{2}|\nabla v|^{2}\right) d x
$$

is decreasing in time. Thus, we are going to consider a fully discrete approximation of the regularized problem (45) using a FE discretization in space and the backward Euler discretization in time (considered for simplicity on a uniform partition of $[0, T]$ with time step $\left.k=T / N:\left(t_{n}=n k\right)_{n=0}^{n=N}\right)$. Let $\Omega$ be a polygonal domain. We consider a shape-regular and quasi-uniform family of triangulations of $\Omega$, denoted by $\left\{\mathcal{T}_{h}\right\}_{h>0}$, with simplices $K, h_{K}=\operatorname{diam}(K)$ and $h:=\max _{K \in \mathcal{T}_{h}} h_{K}$, so that $\bar{\Omega}=\cup_{K \in \mathcal{T}_{h}} \bar{K}$. Further, let $\mathcal{N}_{h}=\left\{\mathbf{a}_{i}\right\}_{i \in \mathcal{I}}$ denote the set of all the vertices of $\mathcal{T}_{h}$, and in this case we will assume the following hypothesis:

(H) The triangulation is structured in the sense that all simplices have a right angle.

We choose the following continuous FE spaces for $u_{\varepsilon}$ and $v_{\varepsilon}$ :

$$
\left(U_{h}, V_{h}\right) \subset H^{1}(\Omega)^{2}, \quad \text { generated by } \mathbb{P}_{1}, \mathbb{P}_{r} \text { with } r \geq 1 \text {. }
$$

Remark 4.2 The right-angled constraint $(\mathbf{H})$ and the approximation of $U_{h}$ by $\mathbb{P}_{1}$ continuous FE are necessary to obtain the relations (49) and (50) below, which are essential in order to obtain the energy-stability of the scheme $\mathbf{U V} \varepsilon$ (see Theorem 4.7 below). 
We denote the Lagrange interpolation operator by $\Pi^{h}: C(\bar{\Omega}) \rightarrow U_{h}$, and we introduce the discrete semi-inner product on $C(\bar{\Omega})$ (which is an inner product in $U_{h}$ ) and its induced discrete seminorm (norm in $U_{h}$ ):

$$
\left(u_{1}, u_{2}\right)^{h}:=\int_{\Omega} \Pi^{h}\left(u_{1} u_{2}\right), \quad|u|_{h}=\sqrt{(u, u)^{h}} .
$$

Remark 4.3 In $U_{h}$, the norms $|\cdot|_{h}$ and $\|\cdot\|_{0}$ are equivalent uniformly with respect to $h[4]$.

We consider also the $L^{2}$-projection on $(\cdot, \cdot)^{h}, Q^{h}: L^{2}(\Omega) \rightarrow U_{h}$ and the classical $H^{1}$-projection $R^{h}: H^{1}(\Omega) \rightarrow V_{h}$ given by

$$
\begin{gathered}
\left(Q^{h} u, \bar{u}\right)^{h}=(u, \bar{u}), \quad \forall \bar{u} \in U_{h}, \\
\left(R^{h} u, \bar{u}\right)+\left(\nabla R^{h} u, \nabla \bar{u}\right)=(u, \bar{u})+(\nabla u, \nabla \bar{u}), \quad \forall \bar{u} \in U_{h} .
\end{gathered}
$$

Moreover, owing to the right-angled constraint $(\mathbf{H})$ and the choice of $\mathbb{P}_{1}$-continuous FE for $U_{h}$, following the ideas of [2] (see also [16]), for each $\varepsilon \in(0,1)$, we can construct two operators $\Lambda_{\varepsilon}^{i}: U_{h} \rightarrow L^{\infty}(\Omega)^{d \times d}(i=1,2)$ such that $\Lambda_{\varepsilon}^{i} u^{h}$ are symmetric matrices and $\Lambda_{\varepsilon}^{1} u^{h}$ is positive definite, for all $u^{h} \in U_{h}$ and a.e. in $\Omega$, and satisfy

$$
\begin{gathered}
\left(\Lambda_{\varepsilon}^{1} u^{h}\right) \nabla \Pi^{h}\left(F_{\varepsilon}^{\prime}\left(u^{h}\right)\right)=\nabla u^{h} \quad \text { in } \Omega, \\
\left(\Lambda_{\varepsilon}^{2} u^{h}\right) \nabla \Pi^{h}\left(F_{\varepsilon}^{\prime}\left(u^{h}\right)\right)=(p-1) \nabla \Pi^{h}\left(F_{\varepsilon}\left(u^{h}\right)\right) \text { in } \Omega .
\end{gathered}
$$

We emphasize that thanks to the choice of $\left\{\mathcal{T}_{h}\right\}_{h>0}$ made up of simplices $K$ (triangles in $2 \mathrm{D}$ and tetrahedra in $3 \mathrm{D}$ ), and the fact that the gradient of a $\mathbb{P}_{1}$-function is constant over each element of the triangular mesh, the operators $\Lambda_{\varepsilon}^{i} u^{h}(i=1,2)$ are constant by elements matrices such that (49) and (50) hold in each element $K$. This condition is not satisfied when rectangular meshes are considered or $\mathbb{P}_{k}$ approximation for $k \geq$ 2. In the 1-dimensional case, $\Lambda_{\varepsilon}^{i}$ are constructed as follows: For all $u^{h} \in U_{h}$ and $K \in \mathcal{T}_{h}$ with vertices $\mathbf{a}_{0}^{K}$ and $\mathbf{a}_{1}^{K}$, we set

$$
\left.\Lambda_{\varepsilon}^{1}\left(u^{h}\right)\right|_{K}:= \begin{cases}\frac{u^{h}\left(\mathbf{a}_{1}^{K}\right)-u^{h}\left(\mathbf{a}_{0}^{K}\right)}{F_{\varepsilon}^{\prime}\left(u^{h}\left(\mathbf{a}_{1}^{K}\right)\right)-F_{\varepsilon}^{\prime}\left(u^{h}\left(\mathbf{a}_{0}^{K}\right)\right)}=\frac{1}{F_{\varepsilon}^{\prime \prime}\left(u^{h}(\xi)\right)} & \text { if } u^{h}\left(\mathbf{a}_{0}^{K}\right) \neq u^{h}\left(\mathbf{a}_{1}^{K}\right), \\ \frac{1}{F_{\varepsilon}^{\prime \prime}\left(u^{h}\left(\mathbf{a}_{0}^{K}\right)\right)} & \text { if } u^{h}\left(\mathbf{a}_{0}^{K}\right)=u^{h}\left(\mathbf{a}_{1}^{K}\right),\end{cases}
$$

for some $\xi \in K$, and

$$
\left.\Lambda_{\varepsilon}^{2}\left(u^{h}\right)\right|_{K}:= \begin{cases}(p-1) \frac{F_{\varepsilon}\left(u^{h}\left(\mathbf{a}_{1}^{K}\right)\right)-F_{\varepsilon}\left(u^{h}\left(\mathbf{a}_{0}^{K}\right)\right)}{F_{\varepsilon}^{\prime}\left(u^{h}\left(\mathbf{a}_{1}^{K}\right)\right)-F_{\varepsilon}^{\prime}\left(u^{h}\left(\mathbf{a}_{0}^{K}\right)\right)}=(p-1) \frac{F_{\varepsilon}^{\prime}\left(u^{h}\left(\xi_{1}\right)\right)}{F_{\varepsilon}^{\prime \prime}\left(u^{h}\left(\xi_{2}\right)\right)} & \text { if } u^{h}\left(\mathbf{a}_{0}^{K}\right) \neq u^{h}\left(\mathbf{a}_{1}^{K}\right), \\ (p-1) \frac{F_{\varepsilon}^{\prime}\left(u^{h}\left(\mathbf{a}_{0}^{K}\right)\right)}{F_{\varepsilon}^{\prime \prime}\left(u^{h}\left(\mathbf{a}_{0}^{K}\right)\right)} & \text { if } u^{h}\left(\mathbf{a}_{0}^{K}\right)=u^{h}\left(\mathbf{a}_{1}^{K}\right),\end{cases}
$$

for some $\xi_{1}, \xi_{2} \in K$. Following [2] (see also [16]), these constructions can be extended to dimensions 2 and 3, and from (51) the following estimate holds:

$$
\varepsilon^{2-p} \xi^{T} \xi \leq \xi^{T} \Lambda_{\varepsilon}^{1}\left(u^{h}\right)^{-1} \xi \leq \varepsilon^{p-2} \xi^{T} \xi, \quad \forall \xi \in \mathbb{R}^{d}, u^{h} \in U_{h}
$$

The following result will be useful to prove the well-posedness of the scheme $\mathbf{U V} \varepsilon$ and we write its proof in the Appendix. 
Lemma 4.4 Let $\|\cdot\|$ denote the spectral norm on $\mathbb{R}^{d \times d}$. Then for any given $\varepsilon \in(0,1)$ the function $\Lambda_{\varepsilon}^{2}: U_{h} \rightarrow\left[L^{\infty}(\Omega)\right]^{d \times d}$ satisfies, for all $u_{1}^{h}, u_{2}^{h} \in U_{h}$ and $K \in \mathcal{T}_{h}$ with vertices $\left\{\mathbf{a}_{l}^{K}\right\}_{l=0}^{d}$,

$$
\begin{aligned}
& \left\|\left(\Lambda_{\varepsilon}^{2}\left(u_{1}^{h}\right)-\Lambda_{\varepsilon}^{2}\left(u_{2}^{h}\right)\right) \mid K\right\| \\
\leq & 3 \varepsilon^{2(p-2)} \max \left\{1,(p-1) \varepsilon^{2(p-2)}\right\} \max _{l=1, \ldots, d}\left\{\mid u_{1}^{h}\left(\mathbf{a}_{l}^{K}\right)-u_{2}^{h}\left(\mathbf{a}_{l}^{K}\right)\right) \mid \\
& \left.+\left|u_{1}^{h}\left(\mathbf{a}_{0}^{K}\right)-u_{2}^{h}\left(\mathbf{a}_{0}^{K}\right)\right|\right\},
\end{aligned}
$$

where $\mathbf{a}_{0}^{K}$ is the right-angled vertex.

Let $A_{h}: V_{h} \rightarrow V_{h}$ be the linear operator defined as follows

$$
\left(A_{h} v^{h}, \bar{v}\right)=\left(\nabla v^{h}, \nabla \bar{v}\right)+\left(v^{h}, \bar{v}\right), \quad \forall \bar{v} \in V_{h} .
$$

Then, the following estimate holds (see for instance, [18, Theorem 3.2]):

$$
\left\|v^{h}\right\|_{W^{1,6}} \leq C\left\|A_{h} v^{h}\right\|_{0}, \quad \forall v^{h} \in V_{h} .
$$

Thus, we consider the following first order in time, nonlinear and coupled scheme:

- $\quad$ Scheme $\boldsymbol{U V \varepsilon}$ :

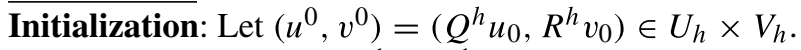

Time step n: Given $\left(u_{\varepsilon}^{n-1}, v_{\varepsilon}^{n-1}\right) \in U_{h} \times V_{h}$, compute $\left(u_{\varepsilon}^{n}, v_{\varepsilon}^{n}\right) \in U_{h} \times V_{h}$ solving

$$
\left\{\begin{array}{l}
\left(\delta_{t} u_{\varepsilon}^{n}, \bar{u}\right)^{h}+\left(\nabla u_{\varepsilon}^{n}, \nabla \bar{u}\right)=-\left(\Lambda_{\varepsilon}^{2}\left(u_{\varepsilon}^{n}\right) \nabla v_{\varepsilon}^{n}, \nabla \bar{u}\right), \quad \forall \bar{u} \in U_{h}, \\
\left(\delta_{t} v_{\varepsilon}^{n}, \bar{v}\right)+\left(A_{h} v_{\varepsilon}^{n}, \bar{v}\right)=p(p-1)\left(\Pi^{h}\left(F_{\varepsilon}\left(u_{\varepsilon}^{n}\right)\right), \bar{v}\right), \quad \forall \bar{v} \in V_{h},
\end{array}\right.
$$

where, in general, we denote $\delta_{t} a^{n}:=\frac{a^{n}-a^{n-1}}{k}$.

Remark 4.5 (Positivity of $v_{\varepsilon}^{n}$ ) By using the mass-lumping technique in all terms of (56) 2 excepting the self-diffusion term $\left(\nabla v_{\varepsilon}^{n}, \nabla \bar{v}\right)$, and approximating $V_{h}$ by $\mathbb{P}_{1-}$ continuous FE, we can prove that if $v_{\varepsilon}^{n-1} \geq 0$ then $v_{\varepsilon}^{n} \geq 0$. In fact, it follows testing $(56)_{2}$ by $\bar{v}=\Pi^{h}\left(v_{\varepsilon-}^{n}\right) \in V_{h}$, where $v_{\varepsilon-}^{n}:=\min \left\{v_{\varepsilon}^{n}, 0\right\}$ (see Remark 3.12 in [20]).

\subsubsection{Mass-conservation, energy-stability and solvability}

Since $\bar{u}=1 \in U_{h}$ and $\bar{v}=1 \in V_{h}$, we deduce that the scheme $\mathbf{U V} \varepsilon$ is conservative in $u_{\varepsilon}^{n}$, that is,

$$
\begin{aligned}
\left(u_{\varepsilon}^{n}, 1\right) & =\left(u_{\varepsilon}^{n}, 1\right)^{h}=\left(u_{\varepsilon}^{n-1}, 1\right)^{h}=\cdots=\left(u^{0}, 1\right)^{h}=\left(u^{0}, 1\right)=\left(Q^{h} u_{0}, 1\right) \\
& =\left(u_{0}, 1\right):=m_{0},
\end{aligned}
$$

and we have the following behavior for $\int_{\Omega} v_{\varepsilon}^{n}$ :

$$
\delta_{t}\left(\int_{\Omega} v_{\varepsilon}^{n}\right)=p(p-1) \int_{\Omega} \Pi^{h}\left(F_{\varepsilon}\left(u_{\varepsilon}^{n}\right)\right)-\int_{\Omega} v_{\varepsilon}^{n} .
$$


Definition 4.6 A numerical scheme with solution $\left(u_{\varepsilon}^{n}, v_{\varepsilon}^{n}\right)$ is called energy-stable with respect to the energy

$$
\mathcal{E}_{\varepsilon}^{h}(u, v)=p\left(F_{\varepsilon}(u), 1\right)^{h}+\frac{1}{2}\|\nabla v\|_{0}^{2}
$$

if this energy is time decreasing, that is $\mathcal{E}_{\varepsilon}^{h}\left(u_{\varepsilon}^{n}, v_{\varepsilon}^{n}\right) \leq \mathcal{E}_{\varepsilon}^{h}\left(u_{\varepsilon}^{n-1}, v_{\varepsilon}^{n-1}\right)$ for all $n \geq 1$.

Theorem 4.7 (Unconditional stability) The scheme $\boldsymbol{U} \boldsymbol{V} \varepsilon$ is unconditionally energy stable with respect to $\mathcal{E}_{\varepsilon}^{h}(u, v)$. In fact, if $\left(u_{\varepsilon}^{n}, v_{\varepsilon}^{n}\right)$ is a solution of $\boldsymbol{U} \boldsymbol{V} \varepsilon$, then the following discrete energy law holds

$$
\begin{aligned}
\delta_{t} \mathcal{E}_{\varepsilon}^{h}\left(u_{\varepsilon}^{n}, v_{\varepsilon}^{n}\right) & +\frac{k \varepsilon^{2-p} p}{2}\left\|\delta_{t} u_{\varepsilon}^{n}\right\|_{0}^{2}+\frac{k}{2}\left\|\delta_{t} \nabla v_{\varepsilon}^{n}\right\|_{0}^{2}+p \varepsilon^{2-p}\left\|\nabla u_{\varepsilon}^{n}\right\|_{0}^{2}+\left\|\left(A_{h}-I\right) v_{\varepsilon}^{n}\right\|_{0}^{2} \\
& +\left\|\nabla v_{\varepsilon}^{n}\right\|_{0}^{2} \leq 0 .
\end{aligned}
$$

Proof Testing $(56)_{1}$ by $\bar{u}=p \Pi^{h}\left(F_{\varepsilon}^{\prime}\left(u_{\varepsilon}^{n}\right)\right)$ and $(56)_{2}$ by $\bar{v}=\left(A_{h}-I\right) v_{\varepsilon}^{n}$, adding and taking into account that $\Lambda_{\varepsilon}^{i}\left(u_{\varepsilon}^{n}\right)$ are symmetric as well as (49) and (50), the terms

$$
\begin{aligned}
-p\left(\Lambda_{\varepsilon}^{2}\left(u_{\varepsilon}^{n}\right) \nabla v_{\varepsilon}^{n}, \nabla \Pi^{h}\left(F_{\varepsilon}^{\prime}\left(u_{\varepsilon}^{n}\right)\right)\right) & =-p\left(\nabla v_{\varepsilon}^{n}, \Lambda_{\varepsilon}^{2}\left(u_{\varepsilon}^{n}\right) \nabla \Pi^{h}\left(F_{\varepsilon}^{\prime}\left(u_{\varepsilon}^{n}\right)\right)\right) \\
& =-p(p-1)\left(\nabla v_{\varepsilon}^{n}, \nabla \Pi^{h}\left(F_{\varepsilon}\left(u_{\varepsilon}^{n}\right)\right)\right)
\end{aligned}
$$

and

$$
p(p-1)\left(\Pi^{h}\left(F_{\varepsilon}\left(u_{\varepsilon}^{n}\right)\right),\left(A_{h}-I\right) v_{\varepsilon}^{n}\right)=p(p-1)\left(\nabla \Pi^{h}\left(F_{\varepsilon}\left(u_{\varepsilon}^{n}\right)\right), \nabla v_{\varepsilon}^{n}\right)
$$

cancel, and using that $\nabla \Pi^{h}\left(F_{\varepsilon}^{\prime}\left(u_{\varepsilon}^{n}\right)\right)=\Lambda_{\varepsilon}^{1}\left(u_{\varepsilon}^{n}\right)^{-1} \nabla u_{\varepsilon}^{n}$ we obtain

$$
\begin{aligned}
p\left(\delta_{t} u_{\varepsilon}^{n}, F_{\varepsilon}^{\prime}\left(u_{\varepsilon}^{n}\right)\right)^{h} & +p \int_{\Omega}\left(\nabla u_{\varepsilon}^{n}\right)^{T} \Lambda_{\varepsilon}^{1}\left(u_{\varepsilon}^{n}\right)^{-1} \nabla u_{\varepsilon}^{n} d \boldsymbol{x} \\
& +\delta_{t}\left(\frac{1}{2}\left\|\nabla v_{\varepsilon}^{n}\right\|_{0}^{2}\right)+\frac{k}{2}\left\|\delta_{t} \nabla v_{\varepsilon}^{n}\right\|_{0}^{2}+\left\|\left(A_{h}-I\right) v_{\varepsilon}^{n}\right\|_{0}^{2} \\
& +\left\|\nabla v_{\varepsilon}^{n}\right\|_{0}^{2}=0 .
\end{aligned}
$$

Moreover, observe that from the Taylor formula we have

$F_{\varepsilon}\left(u_{\varepsilon}^{n-1}\right)=F_{\varepsilon}\left(u_{\varepsilon}^{n}\right)+F_{\varepsilon}^{\prime}\left(u_{\varepsilon}^{n}\right)\left(u_{\varepsilon}^{n-1}-u_{\varepsilon}^{n}\right)+\frac{1}{2} F_{\varepsilon}^{\prime \prime}\left(\theta u_{\varepsilon}^{n}+(1-\theta) u_{\varepsilon}^{n-1}\right)\left(u_{\varepsilon}^{n-1}-u_{\varepsilon}^{n}\right)^{2}$, and therefore,

$$
\delta_{t} u_{\varepsilon}^{n} \cdot F_{\varepsilon}^{\prime}\left(u_{\varepsilon}^{n}\right)=\delta_{t}\left(F_{\varepsilon}\left(u_{\varepsilon}^{n}\right)\right)+\frac{k}{2} F_{\varepsilon}^{\prime \prime}\left(\theta u_{\varepsilon}^{n}+(1-\theta) u_{\varepsilon}^{n-1}\right)\left(\delta_{t} u_{\varepsilon}^{n}\right)^{2} .
$$

Then, using (62) and taking into account that $\Pi^{h}$ is linear and $F_{\varepsilon}^{\prime \prime}(s) \geq \varepsilon^{2-p}$ for all $s \in \mathbb{R}$, we have

$$
\begin{aligned}
\left(\delta_{t} u_{\varepsilon}^{n}, F_{\varepsilon}^{\prime}\left(u_{\varepsilon}^{n}\right)\right)^{h} & =\delta_{t}\left(\int_{\Omega} \Pi^{h}\left(F_{\varepsilon}\left(u_{\varepsilon}^{n}\right)\right)\right)+\frac{k}{2} \int_{\Omega} \Pi^{h}\left(F_{\varepsilon}^{\prime \prime}\left(\theta u_{\varepsilon}^{n}+(1-\theta) u_{\varepsilon}^{n-1}\right)\left(\delta_{t} u_{\varepsilon}^{n}\right)^{2}\right) \\
& \geq \delta_{t}\left(\left(F_{\varepsilon}\left(u_{\varepsilon}^{n}\right), 1\right)^{h}\right)+\frac{k \varepsilon^{2-p}}{2}\left|\delta_{t} u_{\varepsilon}^{n}\right|_{h}^{2} .
\end{aligned}
$$

Thus, from (61), (53), and (63) and Remark 4.3, we arrive at (60). 
Corollary 4.8 (Uniform estimates) Assume that $\left(u_{0}, v_{0}\right) \in L^{2}(\Omega) \times H^{1}(\Omega)$. Let $\left(u_{\varepsilon}^{n}, v_{\varepsilon}^{n}\right)$ be a solution of scheme $\boldsymbol{U V} \varepsilon$. Then, it holds

$$
\begin{aligned}
& p\left(F_{\varepsilon}\left(u_{\varepsilon}^{n}\right), 1\right)^{h}+\frac{1}{2}\left\|v_{\varepsilon}^{n}\right\|_{1}^{2}+k \sum_{m=1}^{n}\left(p \varepsilon^{2-p}\left\|\nabla u_{\varepsilon}^{m}\right\|_{0}^{2}+\left\|\left(A_{h}-I\right) v_{\varepsilon}^{m}\right\|_{0}^{2}+\left\|\nabla v_{\varepsilon}^{m}\right\|_{0}^{2}\right) \\
& \leq C_{0}, \quad \forall n \geq 1, \quad \\
& k \sum_{m=n_{0}+1}^{n+n_{0}}\left\|v_{\varepsilon}^{m}\right\|_{W^{1,6}}^{2} \leq C_{1}(1+k n), \quad \forall n \geq 1,
\end{aligned}
$$

where the integer $n_{0} \geq 0$ is arbitrary, with the constants $C_{0}, C_{1}>0$ depending on the data $\left(\Omega, u_{0}, v_{0}, p\right)$, but independent of $k, h, n$ and $\varepsilon$.

Proof First, taking into account that $\left(u^{0}, v^{0}\right)=\left(Q^{h} u_{0}, R^{h} v_{0}\right), u_{0} \geq 0$ (and therefore, $u^{0} \geq 0$ ), as well as the definition of $F_{\varepsilon}$, we have that

$$
\begin{aligned}
\mathcal{E}_{\varepsilon}^{h}\left(u^{0}, v^{0}\right) & =p \int_{\Omega} \Pi^{h}\left(F_{\varepsilon}\left(u^{0}\right)\right)+\frac{1}{2}\left\|\nabla v^{0}\right\|_{0}^{2} \leq C \int_{\Omega} \Pi^{h}\left(\left(u^{0}\right)^{2}+C\right)+\frac{1}{2}\left\|\nabla v^{0}\right\|_{0}^{2} \\
& \leq C\left(\left\|u^{0}\right\|_{0}^{2}+\left\|\nabla v^{0}\right\|_{0}^{2}+C\right) \leq C\left(\left\|u_{0}\right\|_{0}^{2}+\left\|v_{0}\right\|_{1}^{2}+C\right) \leq C_{0},
\end{aligned}
$$

where the constant $C_{0}>0$ depends on the data $\left(\Omega, u_{0}, v_{0}, p\right)$, but is independent of $k, h, n$ and $\varepsilon$. Therefore, from the discrete energy law (60) and estimate (66), we have $\mathcal{E}_{\varepsilon}^{h}\left(u_{\varepsilon}^{n}, v_{\varepsilon}^{n}\right)+k \sum_{m=1}^{n}\left(p \varepsilon^{2-p}\left\|\nabla u_{\varepsilon}^{m}\right\|_{0}^{2}+\left\|\left(A_{h}-I\right) v_{\varepsilon}^{m}\right\|_{0}^{2}+\left\|\nabla v_{\varepsilon}^{m}\right\|_{0}^{2}\right) \leq \mathcal{E}_{\varepsilon}^{h}\left(u^{0}, v^{0}\right) \leq C_{0}$.

Moreover, from (58), the definition of $F_{\varepsilon}$, Remark 4.11 and (67), we have

$$
(1+k)\left|\int_{\Omega} v_{\varepsilon}^{n}\right|-\left|\int_{\Omega} v_{\varepsilon}^{n-1}\right| \leq k p(p-1) \int_{\Omega} \Pi^{h}\left(F_{\varepsilon}\left(u_{\varepsilon}^{n}\right)\right) \leq k C,
$$

where the constant $C>0$ is independent of $k, h, n$ and $\varepsilon$. Then, applying Lemma 2.2 in (68) (for $\delta=1$ and $\beta=C$ ), we arrive at

$$
\left|\int_{\Omega} v_{\varepsilon}^{n}\right| \leq(1+k)^{-n}\left|\int_{\Omega} v_{h}^{0}\right|+C=(1+k)^{-n}\left|\int_{\Omega} R^{h} v_{0}\right|+C
$$

which, together with (67), imply (64). Moreover, adding (60) from $m=n_{0}+1$ to $m=n+n_{0}$, and using (55) and (64), we deduce (65).

Theorem 4.9 (Unconditional existence) There exists at least one solution $\left(u_{\varepsilon}^{n}, v_{\varepsilon}^{n}\right)$ of scheme $\boldsymbol{U} \boldsymbol{V} \varepsilon$.

Proof The proof follows by using the Leray-Schauder fixed point theorem. With this aim, given $\left(u_{\varepsilon}^{n-1}, v_{\varepsilon}^{n-1}\right) \in U_{h} \times V_{h}$, we define the operator $R: U_{h} \times V_{h} \rightarrow U_{h} \times V_{h}$ 
by $R(\tilde{u}, \widetilde{v})=(u, v)$, such that $(u, v) \in U_{h} \times V_{h}$ solves the following linear decoupled problems

$$
\begin{gathered}
u \in U_{h} \text { s.t. } \frac{1}{k}(u, \bar{u})^{h}+(\nabla u, \nabla \bar{u})=\frac{1}{k}\left(u_{\varepsilon}^{n-1}, \bar{u}\right)^{h}-\left(\Lambda_{\varepsilon}^{2}(\widetilde{u}) \nabla \widetilde{v}, \nabla \bar{u}\right), \quad \forall \bar{u} \in U_{h}, \\
v \in V_{h} \text { s.t. } \frac{1}{k}(v, \bar{v})+\left(A_{h} v, \bar{v}\right)=\frac{1}{k}\left(v_{\varepsilon}^{n-1}, \bar{v}\right)+p(p-1)\left(\Pi^{h}\left(F_{\varepsilon}(\widetilde{u})\right), \bar{v}\right), \quad \forall \bar{v} \in V_{h} .
\end{gathered}
$$

The hypotheses of the Leray-Schauder fixed point theorem are satisfied as in Theorem 3.13 of [20], but applying in this case Lemma 4.4 in order to prove the continuity of the operator $R$. Thus, we conclude that the map $R$ has a fixed point $(u, v)$, that is $R(u, v)=(u, v)$, which is a solution of the scheme $\mathbf{U V} \varepsilon$.

\subsubsection{Approximated positivity of $u_{\varepsilon}^{n}$}

In this subsection we are going to prove the property of approximated positivity for $u_{\varepsilon}^{n}$ solution of the scheme $\mathbf{U} \mathbf{V}_{\varepsilon}$, in the sense that $u_{\varepsilon-}^{n} \rightarrow 0$ as $\varepsilon \rightarrow 0$ in the $L^{2}(\Omega)$ norm, where $u_{\varepsilon-}^{n}:=\min \left\{u_{\varepsilon}^{n}, 0\right\} \leq 0$. With this aim, we will prove first a preliminary result.

Lemma 4.10 The function $\widehat{F}_{\varepsilon}:=F_{\varepsilon}+K(p) \varepsilon^{p}$ (with $\left.K(p)=\frac{(p-2)\left(p^{2}-2 p-1\right)}{2 p(p-1)^{2}}\right)$ satisfies

$$
\widehat{F}_{\varepsilon}(s) \geq \frac{s^{2}}{4 \varepsilon^{2-p}} \quad \forall s \leq \varepsilon \quad \text { and } \quad \widehat{F}_{\varepsilon}(s) \geq C s^{p} \quad \forall s>\varepsilon,
$$

where the constant $C>0$ is independent of $\varepsilon$.

Proof One has that $\widehat{F}_{\varepsilon} \in C^{2}(\mathbb{R})$ since $F_{\varepsilon} \in C^{2}(\mathbb{R})$, and therefore, by using the Taylor formula as well as the definition of $\widehat{F}_{\varepsilon}$ and $\widehat{F}_{\varepsilon}^{\prime}$, we have that, for some $s_{0} \in \mathbb{R}$ between 0 and $s$,

$\widehat{F}_{\varepsilon}(s)=\widehat{F}_{\varepsilon}(0)+\widehat{F}_{\varepsilon}^{\prime}(0) s+\frac{1}{2} \widehat{F}_{\varepsilon}^{\prime \prime}\left(s_{0}\right) s^{2}=\left(\frac{2-p}{p-1}\right)^{2} \varepsilon^{p}+\frac{2-p}{p-1} \varepsilon^{p-1} s+\frac{1}{2} \widehat{F}_{\varepsilon}^{\prime \prime}\left(s_{0}\right) s^{2}$.

Then, taking into account that $\widehat{F}_{\varepsilon}^{\prime \prime}(s)=F_{\varepsilon}^{\prime \prime}(s)=\varepsilon^{p-2}$ for all $s \leq \varepsilon$, from (70) we have that: (a) if $s \in[0, \varepsilon], \widehat{F}_{\varepsilon}(s) \geq \frac{1}{2} \varepsilon^{p-2} s^{2}$; and (b) if $s<0$, by using the Young inequality,

$$
\widehat{F}_{\varepsilon}(s) \geq\left(\frac{2-p}{p-1}\right)^{2} \varepsilon^{p}-\frac{1}{4} \varepsilon^{p-2} s^{2}-\left(\frac{2-p}{p-1}\right)^{2} \varepsilon^{p}+\frac{1}{2} \varepsilon^{p-2} s^{2}=\frac{1}{4} \varepsilon^{p-2} s^{2},
$$

from which we deduce (69) 1 . Finally, (69) 2 follows directly from the definition of $\widehat{F}_{\varepsilon}$ for $s \geq \varepsilon$.

Remark 4.11 Notice that estimates in (69) imply that $|s|^{p} \leq K_{1} \widehat{F}_{\varepsilon}(s)+K_{2}$ for all $s \in \mathbb{R}$, where the constants $K_{1}, K_{2}>0$ are independent of $\varepsilon$. 
Theorem 4.12 (Approximated positivity of $\left.u_{\varepsilon}^{n}\right)$ Let $\left(u_{\varepsilon}^{n}, v_{\varepsilon}^{n}\right)$ be any solution of scheme $\boldsymbol{U} \boldsymbol{V} \varepsilon$. Then, it holds

$$
\max _{n \geq 0}\left\|\Pi^{h}\left(u_{\varepsilon-}^{n}\right)\right\|_{0}^{2} \leq C_{0} \varepsilon^{2-p}\left(\frac{1}{p-1}+\frac{\varepsilon^{p}}{(p-1)^{2}}+1\right) \quad \text { and }\left\|u_{\varepsilon}^{n}\right\|_{L^{p}}^{p} \leq K, \quad \forall n \geq 1,
$$

where the constant $C_{0}$ is independent of $k, h, n, \varepsilon$ and $p$, the constant $K(p)$ was defined in Lemma 4.10, and the constant $K>0$ is independent of $k, h, n$, and $\varepsilon$.

Proof Recall that $\widehat{F}_{\varepsilon}:=F_{\varepsilon}+K(p) \varepsilon^{p}$. Then one can easily verify that (60) remains true for $\widehat{F}_{\varepsilon}\left(u_{\varepsilon}^{n}\right)$ instead of $F_{\varepsilon}\left(u_{\varepsilon}^{n}\right)$ in the term $\delta_{t} \mathcal{E}_{\varepsilon}^{h}\left(u_{\varepsilon}^{n}, v_{\varepsilon}^{n}\right)$. Therefore, arguing as (66) and (67), one has

$$
\begin{array}{r}
p\left(\widehat{F}_{\varepsilon}\left(u_{\varepsilon}^{n}\right), 1\right)^{h}+\frac{1}{2}\left\|\nabla v_{\varepsilon}^{n}\right\|_{0}^{2}+k \sum_{m=1}^{n}\left(p \varepsilon^{2-p}\left\|\nabla u_{\varepsilon}^{m}\right\|_{0}^{2}+\left\|\left(A_{h}-I\right) v_{\varepsilon}^{m}\right\|_{0}^{2}+\left\|\nabla v_{\varepsilon}^{m}\right\|_{0}^{2}\right) \\
\leq C\left(\frac{1}{p-1}\left\|u_{0}\right\|_{L^{p}}^{p}+K(p) \varepsilon^{p}+\left\|\nabla v_{0}\right\|_{0}^{2}+\left\|\left(A_{h}-I\right) v_{0}\right\|_{0}\right), \forall n \geq 1 .(72)
\end{array}
$$

Moreover, from (69) $)_{1}$, we have $\frac{1}{4} \varepsilon^{p-2}\left(u_{\varepsilon-}^{n}(\boldsymbol{x})\right)^{2} \leq \widehat{F}_{\varepsilon}\left(u_{\varepsilon}^{n}(\boldsymbol{x})\right)$ for all $u_{\varepsilon}^{n} \in U_{h}$; and therefore, using that $\left(\Pi^{h} u\right)^{2} \leq \Pi^{h}\left(u^{2}\right)$ for all $u \in C(\bar{\Omega})$, we have

$$
\frac{1}{4} \varepsilon^{p-2} \int_{\Omega}\left(\Pi^{h}\left(u_{\varepsilon-}^{n}\right)\right)^{2} \leq \frac{1}{4} \varepsilon^{p-2} \int_{\Omega} \Pi^{h}\left(\left(u_{\varepsilon-}^{n}\right)^{2}\right) \leq \int_{\Omega} \Pi^{h}\left(\widehat{F}_{\varepsilon}\left(u_{\varepsilon}^{n}\right)\right) .
$$

Thus, from (72) and (73) we obtain that

$$
\max _{n \geq 0}\left\|\Pi^{h}\left(u_{\varepsilon-}^{n}\right)\right\|_{0}^{2} \leq C_{0} \varepsilon^{2-p}\left(\frac{1}{p-1}+|K(p)| \varepsilon^{p}+1\right) .
$$

Since $|K(p)| \leq C /(p-1)^{2}$, we can conclude (71) 1 . Finally, taking into account that $\left|\Pi^{h} u\right|^{p} \leq \Pi^{h}\left(|u|^{p}\right)$ for all $u \in C(\bar{\Omega})$, as well as Remark 4.11 and (64), we have

$$
\left\|u_{\varepsilon}^{n}\right\|_{L^{p}}^{p}=\int_{\Omega}\left|\Pi^{h} u_{\varepsilon}^{n}\right|^{p} \leq \int_{\Omega} \Pi^{h}\left(\left|u_{\varepsilon}^{n}\right|^{p}\right) \leq \int_{\Omega} \Pi^{h}\left(K_{1} \widehat{F}_{\varepsilon}\left(u_{\varepsilon}^{n}\right)+K_{2}\right) \leq K,
$$

arriving at $(71)_{2}$.

Remark 4.13 From $(71)_{1}$ one has that, in order to guarantee the approximated positivity property for the scheme $\mathbf{U V} \varepsilon$, it is necessary to choose $\varepsilon$ such that $\varepsilon^{2-p} /(p-$ $1)+(\varepsilon /(p-1))^{2} \rightarrow 0$ as $\varepsilon \rightarrow 0$.

\subsection{Scheme US $\varepsilon$}

In this section, we are going to construct another energy-stable fully discrete scheme for (3) considering the auxiliary variable $\sigma=\nabla v$ an the regularized function $G_{\varepsilon}(u)=1 / F_{\varepsilon}^{\prime \prime}(u)$. We will also use the regularized functions $F_{\varepsilon}, F_{\varepsilon}^{\prime}$ and $F_{\varepsilon}^{\prime \prime}$ 
defined in Section 4.1. Then, another regularized version of problem (3) reads: Find $u_{\varepsilon}: \Omega \times[0, T] \rightarrow \mathbb{R}$ and $\sigma_{\varepsilon}: \Omega \times[0, T] \rightarrow \mathbb{R}^{d}$, with $u_{\varepsilon} \geq 0$, such that

$$
\left\{\begin{array}{l}
\partial_{t} u_{\varepsilon}-\nabla \cdot\left(G_{\varepsilon}\left(u_{\varepsilon}\right) \nabla\left(F_{\varepsilon}^{\prime}\left(u_{\varepsilon}\right)\right)\right)-\nabla \cdot\left(u_{\varepsilon} \boldsymbol{\sigma}_{\varepsilon}\right)=0 \text { in } \Omega, t>0, \\
\partial_{t} \boldsymbol{\sigma}_{\varepsilon}+\operatorname{rot}\left(\operatorname{rot} \boldsymbol{\sigma}_{\varepsilon}\right)-\nabla\left(\nabla \cdot \boldsymbol{\sigma}_{\varepsilon}\right)+\boldsymbol{\sigma}_{\varepsilon}=p u_{\varepsilon} \nabla\left(F_{\varepsilon}^{\prime}\left(u_{\varepsilon}\right)\right) \text { in } \Omega, t>0, \\
\frac{\partial u_{\varepsilon}}{\partial \mathbf{n}}=0, \quad \boldsymbol{\sigma}_{\varepsilon} \cdot \mathbf{n}=0, \quad\left[\operatorname{rot} \boldsymbol{\sigma}_{\varepsilon} \times \mathbf{n}\right]_{t a n g}=0 \quad \text { on } \partial \Omega, t>0, \\
u_{\varepsilon}(\boldsymbol{x}, 0)=u_{0}(\boldsymbol{x}) \geq 0, \quad \boldsymbol{\sigma}_{\varepsilon}(\boldsymbol{x}, 0)=\nabla v_{0}(\boldsymbol{x}), \quad \text { in } \Omega .
\end{array}\right.
$$

This kind of formulation considering $\sigma=\nabla v$ as auxiliary variable has been used in the construction of numerical schemes for other chemotaxis models (see for instance $[18,20,33])$. Once problem (74) is solved, we can recover $v_{\varepsilon}$ from $u_{\varepsilon}$ by solving

$$
\left\{\begin{array}{l}
\partial_{t} v_{\varepsilon}-\Delta v_{\varepsilon}+v_{\varepsilon}=u_{\varepsilon}^{p} \quad \text { in } \Omega, t>0 \\
\frac{\partial v_{\varepsilon}}{\partial \mathbf{n}}=0 \quad \text { on } \partial \Omega, t>0 \\
v_{\varepsilon}(\boldsymbol{x}, 0)=v_{0}(\boldsymbol{x}) \geq 0 \quad \text { in } \Omega
\end{array}\right.
$$

Observe that (at least formally) multiplying $(74)_{1}$ by $p F_{\varepsilon}^{\prime}\left(u_{\varepsilon}\right),(74)_{2}$ by $\sigma_{\varepsilon}$, integrating over $\Omega$ and adding both equations, the terms $p\left(u_{\varepsilon} \nabla\left(F_{\varepsilon}^{\prime}\left(u_{\varepsilon}\right)\right), \sigma_{\varepsilon}\right)$ cancel, and we obtain the following energy law

$$
\frac{d}{d t} \int_{\Omega}\left(p F_{\varepsilon}\left(u_{\varepsilon}\right)+\frac{1}{2}\left|\boldsymbol{\sigma}_{\varepsilon}\right|^{2}\right) d \boldsymbol{x}+\int_{\Omega} p G_{\varepsilon}\left(u_{\varepsilon}\right)\left|\nabla\left(F_{\varepsilon}^{\prime \prime}\left(u_{\varepsilon}\right)\right)\right|^{2} d \boldsymbol{x}+\left\|\boldsymbol{\sigma}_{\varepsilon}\right\|_{1}^{2}=0 .
$$

In particular, the modified energy $\mathcal{E}_{\varepsilon}(u, \sigma)=\int_{\Omega}\left(p F_{\varepsilon}(u)+\frac{1}{2}|\boldsymbol{\sigma}|^{2}\right) d \boldsymbol{x}$ is decreasing in time. Then, we consider a fully discrete approximation of the regularized problem (74) using a FE discretization in space and the backward Euler discretization in time (considered for simplicity on a uniform partition of $[0, T]$ with time step $k=T / N$ : $\left.\left(t_{n}=n k\right)_{n=0}^{n=N}\right)$. Concerning the space discretization, we consider the triangulation as in the scheme $\mathbf{U V} \varepsilon$, but in this case without imposing the constraint (H) related with the right-angled simplices. We choose the following continuous FE spaces for $u_{\varepsilon}, \sigma_{\varepsilon}$, and $v_{\varepsilon}$ :

$\left(U_{h}, \boldsymbol{\Sigma}_{h}, V_{h}\right) \subset H^{1}(\Omega) \times \mathbf{H}_{\sigma}^{1}(\Omega) \times H^{1}(\Omega), \quad$ generated by $\mathbb{P}_{1}, \mathbb{P}_{m}, \mathbb{P}_{r}$ with $m, r \geq 1$.

Then, we consider the following first order in time, nonlinear and coupled scheme:

- $\quad$ Scheme USE:

Initialization: Let $\left(u^{0}, \boldsymbol{\sigma}^{0}\right)=\left(Q^{h} u_{0}, \widetilde{Q}^{h}\left(\nabla v_{0}\right)\right) \in U_{h} \times \boldsymbol{\Sigma}_{h}$. Here, $Q^{h}$ is the $L^{2}$-projection on $U_{h}$ defined in (48), and $\widetilde{Q}^{h}$ is the classical $L^{2}$-projection on $\boldsymbol{\Sigma}_{h}$,

Time step n: Given $\left(u_{\varepsilon}^{n-1}, \boldsymbol{\sigma}_{\varepsilon}^{n-1}\right) \in U_{h} \times \boldsymbol{\Sigma}_{h}$, compute $\left(u_{\varepsilon}^{n}, \boldsymbol{\sigma}_{\varepsilon}^{n}\right) \in U_{h} \times \boldsymbol{\Sigma}_{h}$ solving

$$
\left\{\begin{array}{l}
\left(\delta_{t} u_{\varepsilon}^{n}, \bar{u}\right)^{h}+\left(G_{\varepsilon}\left(u_{\varepsilon}^{n}\right) \nabla \Pi^{h}\left(F_{\varepsilon}^{\prime}\left(u_{\varepsilon}^{n}\right)\right), \nabla \bar{u}\right)=-\left(\left(u_{\varepsilon}^{n}\right)_{+} \boldsymbol{\sigma}_{\varepsilon}^{n}, \nabla \bar{u}\right), \quad \forall \bar{u} \in U_{h}, \\
\left(\delta_{t} \boldsymbol{\sigma}_{\varepsilon}^{n}, \overline{\boldsymbol{\sigma}}\right)+\left(B_{h} \boldsymbol{\sigma}_{\varepsilon}^{n}, \overline{\boldsymbol{\sigma}}\right)=p\left(\left(u_{\varepsilon}^{n}\right)_{+} \nabla \Pi^{h}\left(F_{\varepsilon}^{\prime}\left(u_{\varepsilon}^{n}\right)\right), \overline{\boldsymbol{\sigma}}\right), \quad \forall \overline{\boldsymbol{\sigma}} \in \Sigma_{h},
\end{array}\right.
$$

where $\left(u_{\varepsilon}^{n}\right)_{+}:=\max \left\{u_{\varepsilon}^{n}, 0\right\} \geq 0$ and the operator $B_{h}$ is defined as

$$
\left(B_{h} \boldsymbol{\sigma}_{\varepsilon}^{n}, \overline{\boldsymbol{\sigma}}\right)=\left(\operatorname{rot} \boldsymbol{\sigma}_{\varepsilon}^{n}, \operatorname{rot} \overline{\boldsymbol{\sigma}}\right)+\left(\nabla \cdot \boldsymbol{\sigma}_{\varepsilon}^{n}, \nabla \cdot \overline{\boldsymbol{\sigma}}\right)+\left(\boldsymbol{\sigma}_{\varepsilon}^{n}, \overline{\boldsymbol{\sigma}}\right), \quad \forall \overline{\boldsymbol{\sigma}} \in \boldsymbol{\Sigma}_{h} .
$$


We recall that $\Pi^{h}: C(\bar{\Omega}) \rightarrow U_{h}$ is the Lagrange interpolation operator, and the discrete semi-inner product $(\cdot, \cdot)^{h}$ was defined in (47).

Once the scheme $\mathbf{U S} \varepsilon$ is solved, given $v_{\varepsilon}^{n-1} \in V_{h}$, we can recover $v_{\varepsilon}^{n}=v_{\varepsilon}^{n}\left(u_{\varepsilon}^{n}\right) \in$ $V_{h}$ solving:

$$
\left(\delta_{t} v_{\varepsilon}^{n}, \bar{v}\right)+\left(\nabla v_{\varepsilon}^{n}, \nabla \bar{v}\right)+\left(v_{\varepsilon}^{n}, \bar{v}\right)=p(p-1)\left(F_{\varepsilon}\left(u_{\varepsilon}^{n}\right), \bar{v}\right), \quad \forall \bar{v} \in V_{h} .
$$

Given $u_{\varepsilon}^{n} \in U_{h}$ and $v_{\varepsilon}^{n-1} \in V_{h}$, Lax-Milgram theorem implies that there exists a unique $v_{\varepsilon}^{n} \in V_{h}$ solution of (76). Moreover, notice that the result concerning to the positivity of $v_{\varepsilon}^{n}$ solution of scheme $\mathbf{U V} \varepsilon$ established in Remark 4.5 remains true for $v_{\varepsilon}^{n}$ in the scheme $\mathbf{U S} \varepsilon$.

\subsubsection{Mass-conservation, energy-stability, solvability and approximated positivity}

Observe that the scheme $\mathbf{U S} \varepsilon$ is also conservative in $u$ (satisfying (57)), and we have the following behavior for $\int_{\Omega} v_{\varepsilon}^{n}$ :

$$
\delta_{t}\left(\int_{\Omega} v_{\varepsilon}^{n}\right)=p(p-1) \int_{\Omega} F_{\varepsilon}\left(u_{\varepsilon}^{n}\right)-\int_{\Omega} v_{\varepsilon}^{n} .
$$

Definition 4.14 A numerical scheme with solution $\left(u_{\varepsilon}^{n}, \boldsymbol{\sigma}_{\varepsilon}^{n}\right)$ is called energy-stable with respect to the energy

$$
\mathcal{E}_{\varepsilon}^{h}(u, \sigma)=p\left(F_{\varepsilon}(u), 1\right)^{h}+\frac{1}{2}\|\boldsymbol{\sigma}\|_{0}^{2}
$$

if this energy is time decreasing, that is $\mathcal{E}_{\varepsilon}^{h}\left(u_{\varepsilon}^{n}, \boldsymbol{\sigma}_{\varepsilon}^{n}\right) \leq \mathcal{E}_{\varepsilon}^{h}\left(u_{\varepsilon}^{n-1}, \boldsymbol{\sigma}_{\varepsilon}^{n-1}\right)$ for all $n \geq 1$.

Theorem 4.15 (Unconditional stability) The scheme $\boldsymbol{U} \boldsymbol{S} \varepsilon$ is unconditionally energy stable with respect to $\mathcal{E}_{\varepsilon}^{h}(u, \boldsymbol{\sigma})$. In fact, if $\left(u_{\varepsilon}^{n}, \boldsymbol{\sigma}_{\varepsilon}^{n}\right)$ is a solution of $\boldsymbol{U} \boldsymbol{S} \varepsilon$, then the following discrete energy law holds

$$
\begin{aligned}
\delta_{t} \mathcal{E}_{\varepsilon}^{h}\left(u_{\varepsilon}^{n}, \boldsymbol{\sigma}_{\varepsilon}^{n}\right) & +\frac{k \varepsilon^{2-p} p}{2}\left\|\delta_{t} u_{\varepsilon}^{n}\right\|_{0}^{2}+\frac{k}{2}\left\|\delta_{t} \boldsymbol{\sigma}_{\varepsilon}^{n}\right\|_{0}^{2}+p \int_{\Omega} G_{\varepsilon}\left(u_{\varepsilon}^{n}\right)\left|\nabla \Pi^{h}\left(F_{\varepsilon}^{\prime}\left(u_{\varepsilon}^{n}\right)\right)\right|^{2} d \boldsymbol{x} \\
& +\left\|\boldsymbol{\sigma}_{\varepsilon}^{n}\right\|_{1}^{2} \leq 0 .
\end{aligned}
$$

Proof Testing $(75)_{1}$ by $\bar{u}=p \Pi^{h}\left(F_{\varepsilon}^{\prime}\left(u_{\varepsilon}^{n}\right)\right),(75)_{2}$ by $\bar{\sigma}=\sigma_{\varepsilon}^{n}$ and adding, the terms $p\left(\left(u_{\varepsilon}^{n}\right)_{+} \nabla \Pi^{h}\left(F_{\varepsilon}^{\prime}\left(u_{\varepsilon}\right)\right), \sigma_{\varepsilon}^{n}\right)$ cancel, and we arrive at

$$
\begin{aligned}
p\left(\delta_{t} u_{\varepsilon}^{n}, F_{\varepsilon}^{\prime}\left(u_{\varepsilon}^{n}\right)\right)^{h} & +p \int_{\Omega} G_{\varepsilon}\left(u_{\varepsilon}^{n}\right)\left|\nabla \Pi^{h}\left(F_{\varepsilon}^{\prime}\left(u_{\varepsilon}^{n}\right)\right)\right|^{2} d \boldsymbol{x}+\delta_{t}\left(\frac{1}{2}\left\|\boldsymbol{\sigma}_{\varepsilon}^{n}\right\|_{0}^{2}\right) \\
& +\frac{k}{2}\left\|\delta_{t} \boldsymbol{\sigma}_{\varepsilon}^{n}\right\|_{0}^{2}+\left\|\boldsymbol{\sigma}_{\varepsilon}^{n}\right\|_{1}^{2}=0,
\end{aligned}
$$

which, proceeding as in (62) and (63) and using Remark 4.3, implies (78). 
Corollary 4.16 (Global energy law) Assume that $\left(u_{0}, v_{0}\right) \in L^{2}(\Omega) \times H^{1}(\Omega)$. Let $\left(u_{\varepsilon}^{n}, \sigma_{\varepsilon}^{n}\right)$ be a solution of scheme $\boldsymbol{U S} \varepsilon$. Then, it holds

$p\left(F_{\varepsilon}\left(u_{\varepsilon}^{n}\right), 1\right)^{h}+\frac{1}{2}\left\|\sigma_{\varepsilon}^{n}\right\|_{0}^{2}+k \sum_{m=1}^{n}\left(p \varepsilon^{2-p}\left\|\nabla \Pi^{h}\left(F_{\varepsilon}^{\prime}\left(u_{\varepsilon}^{m}\right)\right)\right\|_{0}^{2}+\left\|\boldsymbol{\sigma}_{\varepsilon}^{m}\right\|_{1}^{2}\right) \leq C_{0}, \quad \forall n \geq 1$,

with the constant $C_{0}>0$ depending on the data $\left(\Omega, u_{0}, v_{0}, p\right)$, but independent of $k, h, n$ and $\varepsilon$.

Proof Proceeding as in (66) (using the fact that $\left.\left(u^{0}, \sigma^{0}\right)=\left(Q^{h} u_{0}, \widetilde{Q}^{h}\left(\nabla v_{0}\right)\right)\right)$, we can deduce that

$$
p \int_{\Omega} \Pi^{h}\left(F_{\varepsilon}\left(u^{0}\right)\right)+\frac{1}{2}\left\|\sigma^{0}\right\|_{0}^{2} \leq C_{0},
$$

where the constant $C_{0}>0$ depends on the data $\left(\Omega, u_{0}, v_{0}, p\right)$, but is independent of $k, h, n$ and $\varepsilon$. Therefore, from the discrete energy law (78) and estimate (80), we have

$$
\mathcal{E}_{\varepsilon}^{h}\left(u_{\varepsilon}^{n}, \sigma_{\varepsilon}^{n}\right)+k \sum_{m=1}^{n}\left(p \varepsilon^{2-p}\left\|\nabla \Pi^{h}\left(F_{\varepsilon}^{\prime}\left(u_{\varepsilon}^{m}\right)\right)\right\|_{0}^{2}+\left\|\sigma_{\varepsilon}^{m}\right\|_{1}^{2}\right) \leq \mathcal{E}_{\varepsilon}^{h}\left(u^{0}, \sigma^{0}\right) \leq C_{0},
$$

which implies (79).

Remark 4.17 (Approximated positivity of $u_{\varepsilon}^{n}$ ) The approximated positivity result for $u_{\varepsilon}^{n}$ established in Theorem 4.12 remains true for the scheme $\mathbf{U S} \varepsilon$.

Theorem 4.18 (Unconditional solvability) There exists at least one solution $\left(u_{\varepsilon}^{n}, \sigma_{\varepsilon}^{n}\right)$ of scheme $\boldsymbol{U S} \varepsilon$.

Proof The proof follows as in Theorem 4.6 of [20], by using the Leray-Schauder fixed point theorem.

\section{Numerical simulations}

In this section, we will compare the results of several numerical simulations using the schemes derived through the paper. The spaces for $u, \sigma$ and $v$ have been generated by $\mathbb{P}_{1}$-continuous FE, and all the simulations have been carried out using FreeFem++ software. We will also compare with the classical Backward Euler scheme for 
problem (3), which is given for the following first order in time, nonlinear and coupled scheme:

- $\quad$ Scheme $\boldsymbol{U V}$ :

Initialization: Let $\left(u^{0}, v^{0}\right) \in U_{h} \times V_{h}$ an approximation of $\left(u_{0}, v_{0}\right)$ as $h \rightarrow 0$.

Time step n: Given $\left(u^{n-1}, v^{n-1}\right) \in U_{h} \times V_{h}$, compute $\left(u^{n}, v^{n}\right) \in U_{h} \times V_{h}$ by solving

$$
\left\{\begin{array}{l}
\left(\delta_{t} u^{n}, \bar{u}\right)+\left(\nabla u^{n}, \nabla \bar{u}\right)=-\left(u^{n} \nabla v^{n}, \nabla \bar{u}\right), \quad \forall \bar{u} \in U_{h} \\
\left(\delta_{t} v^{n}, \bar{v}\right)+\left(\nabla v^{n}, \nabla \bar{v}\right)+\left(v^{n}, \bar{v}\right)=\left(\left(u_{+}^{n}\right)^{p}, \bar{v}\right), \quad \forall \bar{v} \in V_{h}
\end{array}\right.
$$

Remark 5.1 The scheme UV has not been analyzed in the previous sections because it is not clear how to prove neither its energy-stability nor its approximated positivity. In fact, observe that the scheme $\mathbf{U V} \varepsilon$ (which is the "closest" approximation to the scheme UV considered in this paper) differs from the scheme UV in the use of the regularized function $F_{\varepsilon}$ and its derivatives (see Fig. 1) and in the approximation of the cross-diffusion and production terms, $(u \nabla v, \nabla \bar{u})$ and $\left(u^{p}, \bar{v}\right)$ respectively, which are crucial for the proof of the energy-stability of the scheme $\mathbf{U V} \varepsilon$, and consequently for the approximated positivity.

We have used a structured mesh for the simulations of the scheme $\mathbf{U V} \varepsilon$ (then the right-angled constraint (H) holds), and unstructured meshes for the schemes $\mathbf{U S} \varepsilon$ and UV. The linear iterative methods used to approach the solutions of the nonlinear schemes $\mathbf{U V} \varepsilon, \mathbf{U S} \varepsilon$ and $\mathbf{U V}$ are the following Picard methods:

(i) Picard method to approach a solution $\left(u_{\varepsilon}^{n}, v_{\varepsilon}^{n}\right)$ of the scheme $\mathbf{U V} \varepsilon$ :

Given $\left(u_{\varepsilon}^{l}, v_{\varepsilon}^{l}\right) \in U_{h} \times V_{h}$, compute $\left(u_{\varepsilon}^{l+1}, v_{\varepsilon}^{l+1}\right) \in U_{h} \times V_{h}$ solving the decoupled problems

$$
\begin{aligned}
& \frac{1}{k}\left(u_{\varepsilon}^{l+1}, \bar{u}\right)^{h}+\left(\nabla u_{\varepsilon}^{l+1}, \nabla \bar{u}\right)=\frac{1}{k}\left(u_{\varepsilon}^{n-1}, \bar{u}\right)^{h}-\left(\Lambda_{\varepsilon}^{2}\left(u_{\varepsilon}^{l}\right) \nabla v_{\varepsilon}^{l}, \nabla \bar{u}\right), \quad \forall \bar{u} \in U_{h}, \\
& \frac{1}{k}\left(v_{\varepsilon}^{l+1}, \bar{v}\right)+\left(A_{h} v_{\varepsilon}^{l+1}, \bar{v}\right)=\frac{1}{k}\left(v_{\varepsilon}^{n-1}, \bar{v}\right)+p(p-1)\left(\Pi^{h} F_{\varepsilon}\left(u_{\varepsilon}^{l+1}\right), \bar{v}\right), \quad \forall \bar{v} \in V_{h},
\end{aligned}
$$

and choosing the stopping criterion as $\max \left\{\frac{\left\|u_{\varepsilon}^{l+1}-u_{\varepsilon}^{l}\right\|_{0}}{\left\|u_{\varepsilon}^{l}\right\|_{0}}, \frac{\left\|v_{\varepsilon}^{l+1}-v_{\varepsilon}^{l}\right\|_{0}}{\left\|v_{\varepsilon}^{l}\right\|_{0}}\right\} \leq$ tol.

(ii) Picard method to approach a solution $\left(u_{\varepsilon}^{n}, \boldsymbol{\sigma}_{\varepsilon}^{n}\right)$ of the scheme $\mathbf{U S} \varepsilon$ :

Given $\left(u_{\varepsilon}^{l}, \boldsymbol{\sigma}_{\varepsilon}^{l}\right) \in U_{h} \times \boldsymbol{\Sigma}_{h}$, compute $\left(u_{\varepsilon}^{l+1}, \boldsymbol{\sigma}_{\varepsilon}^{l+1}\right) \in U_{h} \times \boldsymbol{\Sigma}_{h}$ solving the decoupled problems

$$
\begin{aligned}
& \frac{1}{k}\left(u_{\varepsilon}^{l+1}, \bar{u}\right)^{h}+\left(\nabla u_{\varepsilon}^{l+1}, \nabla \bar{u}\right)-\left(\nabla u_{\varepsilon}^{l}, \nabla \bar{u}\right) \\
&= \frac{1}{k}\left(u_{\varepsilon}^{n-1}, \bar{u}\right)^{h}-\left(G_{\varepsilon}\left(u_{\varepsilon}^{l}\right) \nabla \Pi^{h}\left(F_{\varepsilon}^{\prime}\left(u_{\varepsilon}^{l}\right)\right), \nabla \bar{u}\right)-\left(\left(u_{\varepsilon}^{l}\right)_{+} \boldsymbol{\sigma}_{\varepsilon}^{l}, \nabla \bar{u}\right), \quad \forall \bar{u} \in U_{h}, \\
& \frac{1}{k}\left(\boldsymbol{\sigma}_{\varepsilon}^{l+1}, \overline{\boldsymbol{\sigma}}\right)+\left(B_{h} \boldsymbol{\sigma}_{\varepsilon}^{l+1}, \overline{\boldsymbol{\sigma}}\right)=\frac{1}{k}\left(\boldsymbol{\sigma}_{\varepsilon}^{n-1}, \overline{\boldsymbol{\sigma}}\right)+p\left(\left(u_{\varepsilon}^{l+1}\right)_{+} \nabla \Pi^{h}\left(F_{\varepsilon}^{\prime}\left(u_{\varepsilon}^{l+1}\right)\right), \overline{\boldsymbol{\sigma}}\right), \\
& \forall \overline{\boldsymbol{\sigma}} \in \Sigma_{h},
\end{aligned}
$$

choosing the stopping criterion $\max \left\{\frac{\left\|u_{\varepsilon}^{l+1}-u_{\varepsilon}^{l}\right\|_{0}}{\left\|u_{\varepsilon}^{l}\right\|_{0}}, \frac{\left\|\sigma_{\varepsilon}^{l+1}-\sigma_{\varepsilon}^{l}\right\|_{0}}{\left\|\sigma_{\varepsilon}^{l}\right\|_{0}}\right\} \leq$ tol. Note that a residual term $\left(\nabla\left(u_{\varepsilon}^{l+1}-u_{\varepsilon}^{l}\right), \nabla \bar{u}\right)$ is considered. This term is 
required in order to improve the convergence of this iterative method. Indeed, since the self-diffusion term of the u-equation is rewritten in a nonlinear form, we have checked that this fact makes the convergence of the corresponding iterative method worse.

(iii) Picard method to approach a solution $\left(u^{n}, v^{n}\right)$ of the scheme $\mathbf{U V}$ :

Given $\left(u^{l}, v^{l}\right) \in U_{h} \times V_{h}$, compute $\left(u^{l+1}, v^{l+1}\right) \in U_{h} \times V_{h}$ solving the decoupled problems

$$
\begin{aligned}
& \frac{1}{k}\left(u^{l+1}, \bar{u}\right)+\left(\nabla u^{l+1}, \nabla \bar{u}\right)+\left(u^{l+1} \nabla v^{l}, \nabla \bar{u}\right)=\frac{1}{k}\left(u^{n-1}, \bar{u}\right), \quad \forall \bar{u} \in U_{h}, \\
& \frac{1}{k}\left(v^{l+1}, \bar{v}\right)+\left(\nabla v^{l+1}, \nabla \bar{v}\right)+\left(v^{l+1}, \bar{v}\right)=\frac{1}{k}\left(v^{n-1}, \bar{v}\right)+\left(\left(u_{+}^{l+1}\right)^{p}, \bar{v}\right), \quad \forall \bar{v} \in V_{h},
\end{aligned}
$$

and choosing the stopping criterion $\max \left\{\frac{\left\|u^{l+1}-u^{l}\right\|_{0}}{\left\|u^{l}\right\|_{0}}, \frac{\left\|v^{l+1}-v^{l}\right\|_{0}}{\left\|v^{l}\right\|_{0}}\right\} \leq$ tol.

Remark 5.2 In all cases, first we compute $u^{l+1}$ solving the $u$-equation, and then, inserting $u^{l+1}$ in the $v$-equation (resp. $\sigma$-system), we compute $v^{l+1}$ (resp. $\sigma^{l+1}$ ).

\subsection{Positivity of $u^{n}$}

In this subsection, the positivity of the variable $u^{n}$ in the three schemes is compared. We recall that for the two schemes studied in this paper, namely schemes $\mathbf{U V} \varepsilon$ and $\mathbf{U S} \varepsilon$, the positivity of the variable $u^{n}$ is not clear. However, it was proved that $\Pi^{h}\left[\left(u_{\varepsilon}^{n}\right)_{-}\right] \rightarrow 0$ as $\varepsilon \rightarrow 0$ (see Theorem 4.12 and Remark 4.17). For this reason, in Figs. 3, 4 and 5 we compare the positivity of the variable $u_{\varepsilon}^{n}$ in the schemes, for different values of $p, 1<p<2$, and taking $\varepsilon=10^{-5}$ and $\varepsilon=10^{-8}$. We consider $\Omega=(0,2)^{2}, k=10^{-5}, h=\frac{1}{80}$, the tolerance parameter $t o l=10^{-4}$ and the initial conditions (see Fig. 2)

$$
\begin{gathered}
u_{0}=-10 x y(2-x)(2-y) \exp \left(-10(y-1)^{2}-10(x-1)^{2}\right)+10.01 \\
v_{0}=80 x y(2-x)(2-y) \exp \left(-30(y-1)^{2}-30(x-1)^{2}\right)+0.01
\end{gathered}
$$

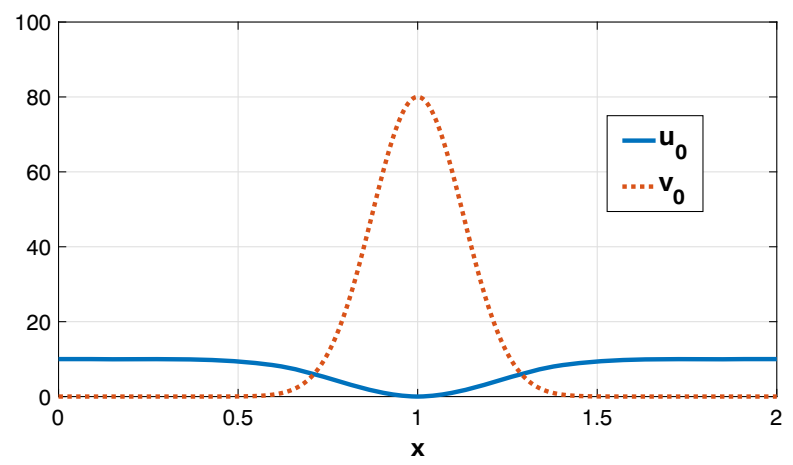

Fig. 2 Cross section at $y=1$ of the initial cell density $u_{0}$ and chemical concentration $v_{0}$ 


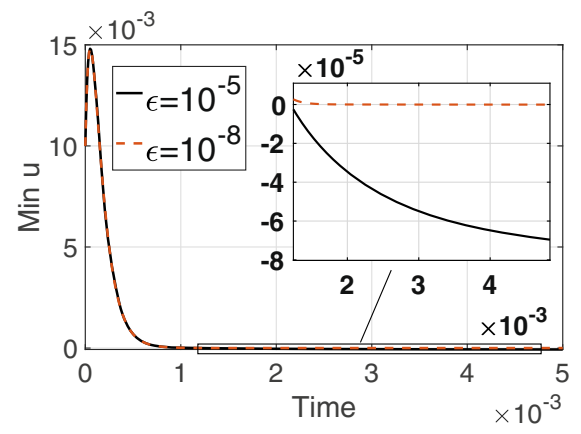

(a) Scheme UVE

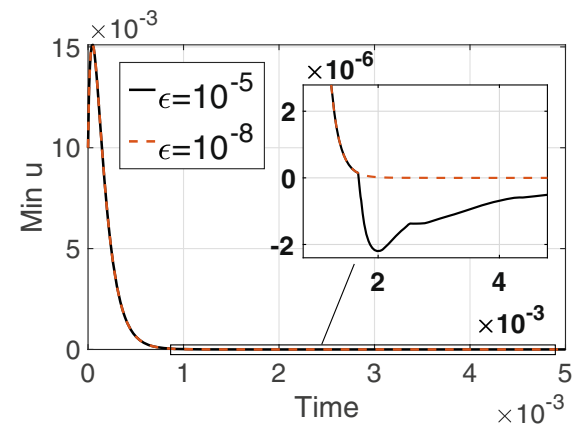

(b) Scheme $\mathbf{U S} \varepsilon$

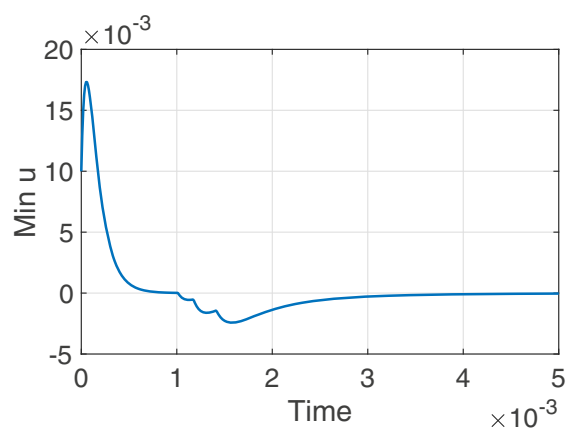

(c) Scheme UV

Fig. 3 Behavior of the minimum of $u^{n}$, taking $p=1.1$

Note that $u_{0}, v_{0}>0$ in $\Omega, \min \left(u_{0}\right)=u_{0}(1,1)=0.01$ and $\max \left(v_{0}\right)=v_{0}(1,1)=$ 80.01. We obtain that:

(i) All the schemes take negative values for the minimum of $u^{n}$ in different times $t_{n} \geq 0$, for the different values considered for $p$ and $\varepsilon$. However, in the case of the schemes $\mathbf{U V} \varepsilon$ and $\mathbf{U S} \varepsilon$, it is observed that these values are closer to 0 as $\varepsilon \rightarrow 0$ (see Figs. 3, 4, and 5).

(ii) In the cases $p=1.1$ y $p=1.5$, the scheme $\mathbf{U S} \varepsilon$ "preserves" better the positivity than the other schemes; while for $p=1.9$, the scheme $\mathbf{U V} \varepsilon$ evidence "better" the positivity (see Figs. 3, 4, and 5).

\subsection{Energy-stability}

In this subsection, we compare numerically the stability of the schemes $\mathbf{U V} \varepsilon, \mathbf{U S} \varepsilon$ and UV with respect to the "exact" energy

$$
\mathcal{E}_{e}(u, v)=\int_{\Omega} \frac{1}{p}\left(u_{+}\right)^{p} d \boldsymbol{x}+\frac{p-1}{2 p}\|\nabla v\|_{0}^{2} .
$$


It was proved that the schemes $\mathbf{U V} \varepsilon$ and $\mathbf{U S} \varepsilon$ are unconditionally energy-stables with respect to modified energies defined in terms of the variables of each scheme, and some energy inequalities are satisfied (see Theorems 4.7 and 4.15). However, it is not clear how to prove the energy-stability of these schemes with respect to the "exact" energy $\mathcal{E}_{e}(u, v)$ given in (81), which comes from the continuous problem (3) (see (8) and (9)). Therefore, it is interesting to compare numerically the schemes with respect to this energy $\mathcal{E}_{e}(u, v)$, and to study the behavior of the following "residual" of the discrete energy law

$$
R E_{e}^{n}:=\delta_{t} \mathcal{E}_{e}\left(u^{n}, v^{n}\right)+\frac{4(p-1)}{p^{2}} \int_{\Omega}\left|\nabla\left(\left(u_{+}^{n}\right)^{p / 2}\right)\right|^{2} d x+\frac{p-1}{p}\left(\left\|\Delta_{h} v^{n}\right\|_{0}^{2}+\left\|\nabla v^{n}\right\|_{0}^{2}\right) .
$$

We consider $\Omega=(0,2)^{2}, k=10^{-5}, h=\frac{1}{25}, p=1.4$, tol $=10^{-4}$ and the initial conditions (see Fig. 6)

$u_{0}=14 \cos (2 \pi x) \cos (2 \pi y)+14.0001$ and $v_{0}=-14 \cos (2 \pi x) \cos (2 \pi y)+14.0001$.

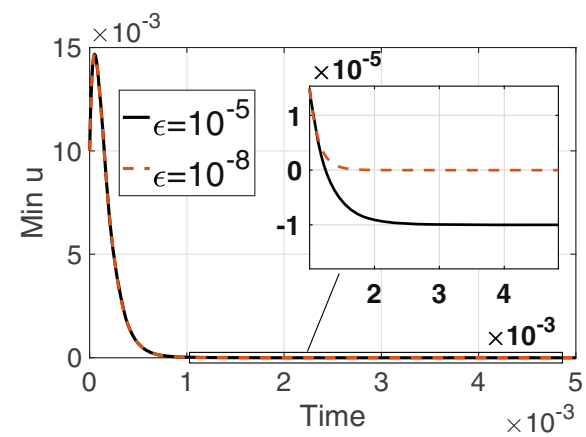

(a) Scheme UV $\varepsilon$

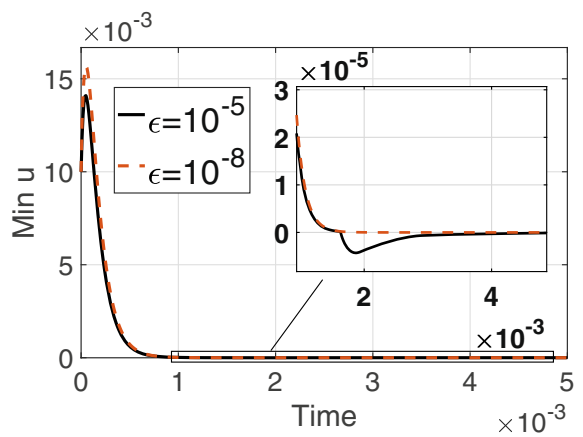

(b) Scheme $\mathbf{U S} \varepsilon$

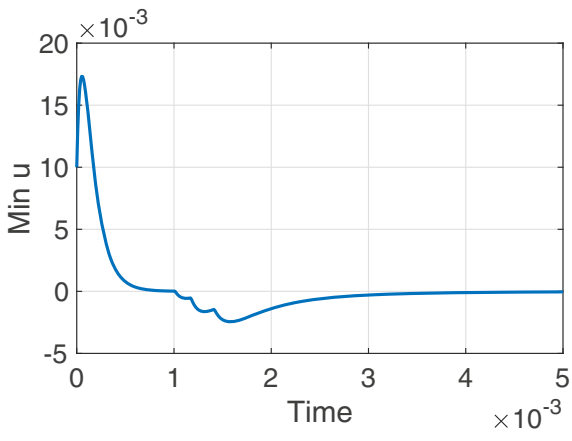

(c) Scheme UV

Fig. 4 Behavior of the minimum of $u^{n}$, taking $p=1.5$ 


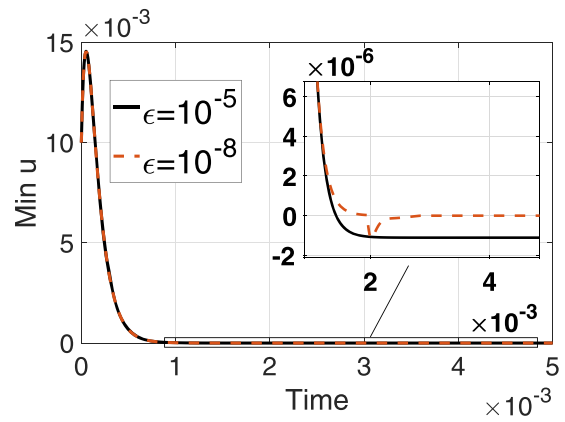

(a) Scheme UV $\varepsilon$

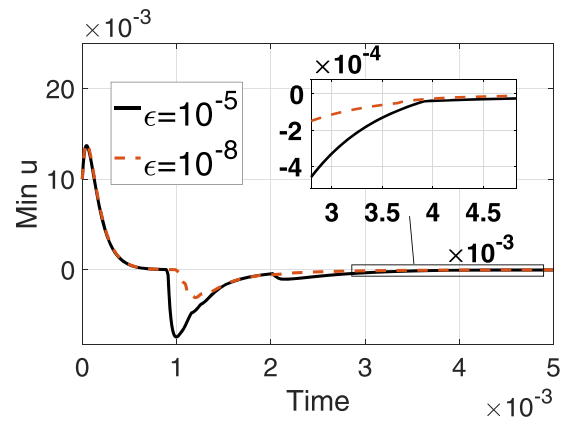

(b) Scheme US $\varepsilon$

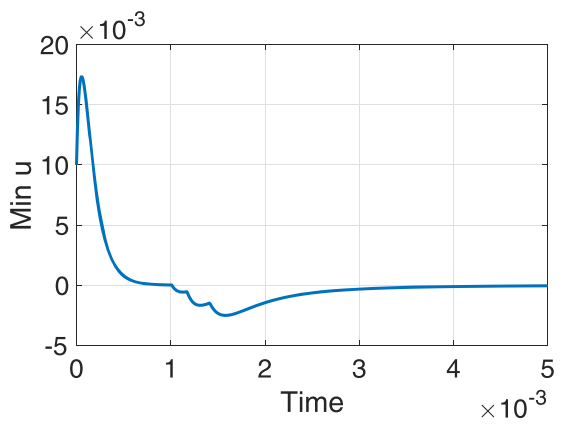

(c) Scheme UV

Fig. 5 Behavior of the minimum of $u^{n}$, taking $p=1.9$

Then, we obtain that:

(i) All the schemes $\mathbf{U V} \varepsilon, \mathbf{U S} \varepsilon$ and $\mathbf{U V}$ satisfy the energy decreasing in time property for the exact energy $\mathcal{E}_{e}(u, v)$ (see Fig. 7a), that is,

$$
\mathcal{E}_{e}\left(u^{n}, v^{n}\right) \leq \mathcal{E}_{e}\left(u^{n-1}, v^{n-1}\right) \forall n .
$$

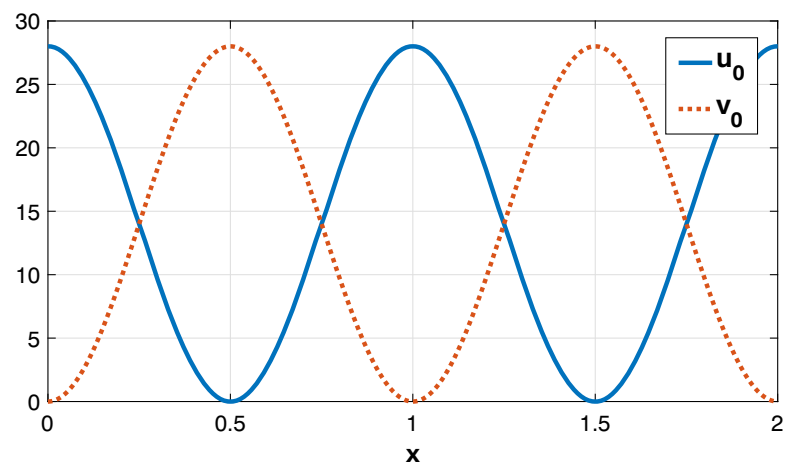

Fig. 6 Cross section at $y=0$ of the initial cell density $u_{0}$ and chemical concentration $v_{0}$ 


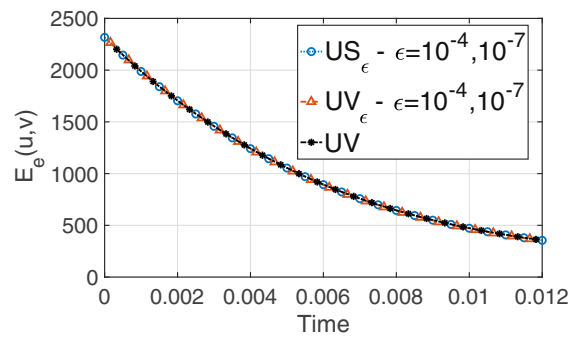

(a) Behaviour of the exact energy $\mathcal{E}_{e}(u, v)$.

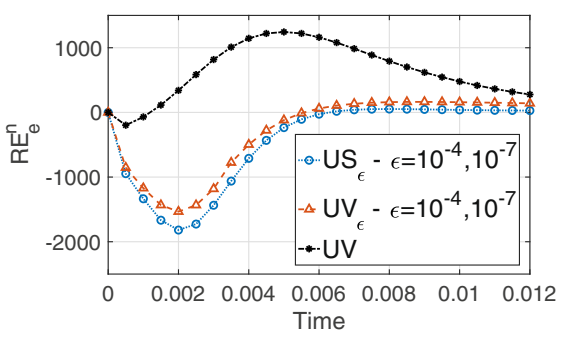

(b) Behaviour of the discrete residual $R E_{e}^{n}$.

Fig. 7 Energy-stability with respect to the exact energy $\mathcal{E}_{e}(u, v)$

(ii) All the schemes show $R E_{e}^{n}>0$ for some $t_{n} \geq 0$; being those corresponding to scheme $\mathbf{U V}$ that reach higher values, while the scheme $\mathbf{U S} \mathbf{S}_{\varepsilon}$ evidence the smallest values. Moreover, it is observed that the scheme $\mathbf{U V} \varepsilon$ introduces lower numerical source than the scheme $\mathbf{U V}$, and lower numerical dissipation than the scheme $\mathbf{U S} \varepsilon$ (see Fig. 7b).

\subsection{Experimental convergence rates}

In order to show the accuracy of the schemes proposed in this paper, we compare the schemes $\mathbf{U} \mathbf{V}_{\varepsilon}, \mathbf{U S}_{\varepsilon}$ and $\mathbf{U V}$ against an exact solution and on several meshes. With this aim, in this experiment we consider the exact solution

$$
\begin{gathered}
u=e^{-t}(\cos (2 \pi x) \cos (2 \pi y)+2), \\
v=(1+\sin (t))(\cos (2 \pi x) \cos (2 \pi y)+2) .
\end{gathered}
$$

Note that $\frac{\partial u}{\partial \boldsymbol{n}}=\frac{\partial v}{\partial \boldsymbol{n}}=0$ on $\partial \Omega$. Moreover, we use a uniform partition with $m+1$ nodes in each direction. We consider $\Omega=(0,1)^{2}$ and $\varepsilon=10^{-6}$.

Numerical results of convergence rates in space are listed in Tables 1, 2, 3, 4, 5, 6, 7, 8 and 9 for $\Delta t=5 \times 10^{-5}$ with respect to the final time $T=0.1$. We denote the total errors by $e_{u}^{n}:=u\left(t_{n}\right)-u_{\varepsilon}^{n}$ and $e_{v}^{n}:=v\left(t_{n}\right)-v_{\varepsilon}^{n}$. For the three schemes $\mathbf{U V} \mathbf{V}_{\varepsilon}, \mathbf{U S}_{\varepsilon}$ and $\mathbf{U V}$, and different values of $p$, we obtain optimal order of convergence in space, that is, second-order for $e_{u}^{n}, e_{v}^{n}$ in $l^{\infty}\left(L^{2}\right)$-norm and first order in $l^{2}\left(H^{1}\right)$-norm.

Table 1 Convergence rates for $p=1.1$ in the scheme $\mathbf{U} \mathbf{V}_{\varepsilon}$

\begin{tabular}{lllllllll}
\hline$m \times m$ & $\left\|e_{u}^{n}\right\|_{l^{\infty}\left(L^{2}\right)}$ & Order & $\left\|e_{u}^{n}\right\|_{l^{2}\left(H^{1}\right)}$ & Order & $\left\|e_{v}^{n}\right\|_{l^{\infty}\left(L^{2}\right)}$ & Order & $\left\|e_{v}^{n}\right\|_{l^{2}\left(H^{1}\right)}$ & Order \\
\hline $36 \times 36$ & $4.8694 \mathrm{e}-03$ & - & $1.1647 \mathrm{e}-01$ & - & $4.8717 \mathrm{e}-03$ & - & $1.2842 \mathrm{e}-01$ & - \\
$44 \times 44$ & $3.2658 \mathrm{e}-03$ & 1.9907 & $9.5368 \mathrm{e}-02$ & 0.9961 & $3.2717 \mathrm{e}-03$ & 1.9841 & $1.0517 \mathrm{e}-01$ & 0.9951 \\
$52 \times 52$ & $2.3386 \mathrm{e}-03$ & 1.9989 & $8.0732 \mathrm{e}-02$ & 0.9973 & $2.3483 \mathrm{e}-03$ & 1.9850 & $8.9042 \mathrm{e}-02$ & 0.9966 \\
$60 \times 60$ & $1.7551 \mathrm{e}-03$ & 2.0058 & $6.9988 \mathrm{e}-02$ & 0.9980 & $1.7678 \mathrm{e}-03$ & 1.9842 & $7.7198 \mathrm{e}-02$ & 0.9974 \\
\hline
\end{tabular}


Table 2 Convergence rates for $p=1.5$ in the scheme $\mathbf{U V} \mathbf{V}_{\varepsilon}$

\begin{tabular}{lllllllll}
\hline$m \times m$ & $\left\|e_{u}^{n}\right\|_{l^{\infty}\left(L^{2}\right)}$ & Order & $\left\|e_{u}^{n}\right\|_{l^{2}\left(H^{1}\right)}$ & Order & $\left\|e_{v}^{n}\right\|_{l^{\infty}\left(L^{2}\right)}$ & Order & $\left\|e_{v}^{n}\right\|_{l^{2}\left(H^{1}\right)}$ & Order \\
\hline $36 \times 36$ & $4.9220 \mathrm{e}-03$ & - & $1.1647 \mathrm{e}-01$ & - & $4.9150 \mathrm{e}-03$ & - & $1.2842 \mathrm{e}-01$ & - \\
$44 \times 44$ & $3.3010 \mathrm{e}-03$ & 1.9907 & $9.5368 \mathrm{e}-02$ & 0.9961 & $3.3009 \mathrm{e}-03$ & 1.9839 & $1.0517 \mathrm{e}-01$ & 0.9951 \\
$52 \times 52$ & $2.3638 \mathrm{e}-03$ & 1.9990 & $8.0732 \mathrm{e}-02$ & 0.9973 & $2.3693 \mathrm{e}-03$ & 1.9849 & $8.9042 \mathrm{e}-02$ & 0.9966 \\
$60 \times 60$ & $1.7740 \mathrm{e}-03$ & 2.0059 & $6.9988 \mathrm{e}-02$ & 0.9980 & $1.7836 \mathrm{e}-03$ & 1.9842 & $7.7198 \mathrm{e}-02$ & 0.9974 \\
\hline
\end{tabular}

Table 3 Convergence rates for $p=1.9$ in the scheme $\mathbf{U} \mathbf{V}_{\varepsilon}$

\begin{tabular}{lllllllll}
\hline$m \times m$ & $\left\|e_{u}^{n}\right\|_{l^{\infty}\left(L^{2}\right)}$ & Order & $\left\|e_{u}^{n}\right\|_{l^{2}\left(H^{1}\right)}$ & Order & $\left\|e_{v}^{n}\right\|_{l^{\infty}\left(L^{2}\right)}$ & Order & $\left\|e_{v}^{n}\right\|_{l^{2}\left(H^{1}\right)}$ & Order \\
\hline $36 \times 36$ & $4.9552 \mathrm{e}-03$ & - & $1.1647 \mathrm{e}-01$ & - & $4.9785 \mathrm{e}-03$ & - & $1.2842 \mathrm{e}-01$ & - \\
$44 \times 44$ & $3.3232 \mathrm{e}-03$ & 1.9908 & $9.5368 \mathrm{e}-02$ & 0.9962 & $3.3437 \mathrm{e}-03$ & 1.9836 & $1.0517 \mathrm{e}-01$ & 0.9951 \\
$52 \times 52$ & $2.3797 \mathrm{e}-03$ & 1.9991 & $8.0732 \mathrm{e}-02$ & 0.9973 & $2.4001 \mathrm{e}-03$ & 1.9846 & $8.9042 \mathrm{e}-02$ & 0.9966 \\
$60 \times 60$ & $1.7859 \mathrm{e}-03$ & 2.0060 & $6.9988 \mathrm{e}-02$ & 0.9980 & $1.8069 \mathrm{e}-03$ & 1.9839 & $7.7198 \mathrm{e}-02$ & 0.9974 \\
\hline
\end{tabular}

Table 4 Convergence rates for $p=1.1$ in the scheme $\mathbf{U S}_{\varepsilon}$

\begin{tabular}{lllllllll}
\hline$m \times m$ & $\left\|e_{u}^{n}\right\|_{l^{\infty}\left(L^{2}\right)}$ & Order & $\left\|e_{u}^{n}\right\|_{l^{2}\left(H^{1}\right)}$ & Order & $\left\|e_{v}^{n}\right\|_{l^{\infty}\left(L^{2}\right)}$ & Order & $\left\|e_{v}^{n}\right\|_{l^{2}\left(H^{1}\right)}$ & Order \\
\hline $36 \times 36$ & $4.1857 \mathrm{e}-03$ & - & $1.1821 \mathrm{e}-01$ & - & $4.7592 \mathrm{e}-03$ & - & $1.2842 \mathrm{e}-01$ & - \\
$44 \times 44$ & $2.8272 \mathrm{e}-03$ & 1.9554 & $9.6354 \mathrm{e}-02$ & 1.0191 & $3.1958 \mathrm{e}-03$ & 1.9847 & $1.0517 \mathrm{e}-01$ & 0.9951 \\
$52 \times 52$ & $2.0386 \mathrm{e}-03$ & 1.9578 & $8.1350 \mathrm{e}-02$ & 1.0133 & $2.2937 \mathrm{e}-03$ & 1.9854 & $8.9043 \mathrm{e}-02$ & 0.9966 \\
$60 \times 60$ & $1.5410 \mathrm{e}-03$ & 1.9553 & $7.0407 \mathrm{e}-02$ & 1.0096 & $1.7267 \mathrm{e}-03$ & 1.9844 & $7.7199 \mathrm{e}-02$ & 0.9974 \\
\hline
\end{tabular}

Table 5 Convergence rates for $p=1.5$ in the scheme $\mathbf{U S}_{\varepsilon}$

\begin{tabular}{lllllllll}
\hline$m \times m$ & $\left\|e_{u}^{n}\right\|_{l^{\infty}\left(L^{2}\right)}$ & Order & $\left\|e_{u}^{n}\right\|_{l^{2}\left(H^{1}\right)}$ & Order & $\left\|e_{v}^{n}\right\|_{l^{\infty}\left(L^{2}\right)}$ & Order & $\left\|e_{v}^{n}\right\|_{l^{2}\left(H^{1}\right)}$ & Order \\
\hline $36 \times 36$ & $4.2616 \mathrm{e}-03$ & - & $1.1824 \mathrm{e}-01$ & - & $4.7199 \mathrm{e}-03$ & - & $1.2842 \mathrm{e}-01$ & - \\
$44 \times 44$ & $2.8767 \mathrm{e}-03$ & 1.9584 & $9.6365 \mathrm{e}-02$ & 1.0194 & $3.1691 \mathrm{e}-03$ & 1.9850 & $1.0517 \mathrm{e}-01$ & 0.9951 \\
$52 \times 52$ & $2.0735 \mathrm{e}-03$ & 1.9599 & $8.1357 \mathrm{e}-02$ & 1.0134 & $2.2744 \mathrm{e}-03$ & 1.9857 & $8.9043 \mathrm{e}-02$ & 0.9966 \\
$60 \times 60$ & $1.5670 \mathrm{e}-03$ & 1.9570 & $7.0411 \mathrm{e}-02$ & 1.0097 & $1.7121 \mathrm{e}-03$ & 1.9848 & $7.7199 \mathrm{e}-02$ & 0.9975 \\
\hline
\end{tabular}

Table 6 Convergence rates for $p=1.9$ in the scheme $\mathbf{U S}_{\varepsilon}$

\begin{tabular}{lllllllll}
\hline$m \times m$ & $\left\|e_{u}^{n}\right\|_{l^{\infty}\left(L^{2}\right)}$ & Order & $\left\|e_{u}^{n}\right\|_{l^{2}\left(H^{1}\right)}$ & Order & $\left\|e_{v}^{n}\right\|_{l^{\infty}\left(L^{2}\right)}$ & Order & $\left\|e_{v}^{n}\right\|_{l^{2}\left(H^{1}\right)}$ & Order \\
\hline $36 \times 36$ & $4.2053 \mathrm{e}-03$ & - & $1.1820 \mathrm{e}-01$ & - & $4.6755 \mathrm{e}-03$ & - & $1.2842 \mathrm{e}-01$ & - \\
$44 \times 44$ & $2.8380 \mathrm{e}-03$ & 1.9597 & $9.6345 \mathrm{e}-02$ & 1.0189 & $3.1390 \mathrm{e}-03$ & 1.9854 & $1.0517 \mathrm{e}-01$ & 0.9951 \\
$52 \times 52$ & $2.0453 \mathrm{e}-03$ & 1.9607 & $8.1345 \mathrm{e}-02$ & 1.0131 & $2.2527 \mathrm{e}-03$ & 1.9860 & $8.9043 \mathrm{e}-02$ & 0.9966 \\
$60 \times 60$ & $1.5457 \mathrm{e}-03$ & 1.9574 & $7.0403 \mathrm{e}-02$ & 1.0095 & $1.6957 \mathrm{e}-03$ & 1.9849 & $7.7199 \mathrm{e}-02$ & 0.9975 \\
\hline
\end{tabular}


Table 7 Convergence rates for $p=1.1$ in the scheme $\mathbf{U V}$

\begin{tabular}{lllllllll}
\hline$m \times m$ & $\left\|e_{u}^{n}\right\|_{l^{\infty}\left(L^{2}\right)}$ & Order & $\left\|e_{u}^{n}\right\|_{l^{2}\left(H^{1}\right)}$ & Order & $\left\|e_{v}^{n}\right\|_{l^{\infty}\left(L^{2}\right)}$ & Order & $\left\|e_{v}^{n}\right\|_{l^{2}\left(H^{1}\right)}$ & Order \\
\hline $36 \times 36$ & $5.1255 \mathrm{e}-03$ & - & $1.1650 \mathrm{e}-01$ & - & $4.8769 \mathrm{e}-03$ & - & $1.2842 \mathrm{e}-01$ & - \\
$44 \times 44$ & $3.4248 \mathrm{e}-03$ & 2.0091 & $9.5385 \mathrm{e}-02$ & 0.9967 & $3.2750 \mathrm{e}-03$ & 1.9843 & $1.0517 \mathrm{e}-01$ & 0.9951 \\
$52 \times 52$ & $2.4425 \mathrm{e}-03$ & 2.0236 & $8.0742 \mathrm{e}-02$ & 0.9976 & $2.3505 \mathrm{e}-03$ & 1.9854 & $8.9042 \mathrm{e}-02$ & 0.9966 \\
$60 \times 60$ & $1.8246 \mathrm{e}-03$ & 2.0381 & $6.9994 \mathrm{e}-02$ & 0.9982 & $1.7694 \mathrm{e}-03$ & 1.9848 & $7.7198 \mathrm{e}-02$ & 0.9974 \\
\hline
\end{tabular}

Table 8 Convergence rates for $p=1.5$ in the scheme $\mathbf{U V}$

\begin{tabular}{lllllllll}
\hline$m \times m$ & $\left\|e_{u}^{n}\right\|_{l^{\infty}\left(L^{2}\right)}$ & Order & $\left\|e_{u}^{n}\right\|_{l^{2}\left(H^{1}\right)}$ & Order & $\left\|e_{v}^{n}\right\|_{l^{\infty}\left(L^{2}\right)}$ & Order & $\left\|e_{v}^{n}\right\|_{l^{2}\left(H^{1}\right)}$ & Order \\
\hline $36 \times 36$ & $5.0897 \mathrm{e}-03$ & - & $1.1650 \mathrm{e}-01$ & - & $4.9202 \mathrm{e}-03$ & - & $1.2842 \mathrm{e}-01$ & - \\
$44 \times 44$ & $3.4011 \mathrm{e}-03$ & 2.0089 & $9.5384 \mathrm{e}-02$ & 0.9966 & $3.3041 \mathrm{e}-03$ & 1.9843 & $1.0517 \mathrm{e}-01$ & 0.9951 \\
$52 \times 52$ & $2.4257 \mathrm{e}-03$ & 2.0232 & $8.0742 \mathrm{e}-02$ & 0.9976 & $2.3714 \mathrm{e}-03$ & 1.9855 & $8.9042 \mathrm{e}-02$ & 0.9966 \\
$60 \times 60$ & $1.8122 \mathrm{e}-03$ & 2.0375 & $6.9994 \mathrm{e}-02$ & 0.9982 & $1.7850 \mathrm{e}-03$ & 1.9850 & $7.7198 \mathrm{e}-02$ & 0.9974 \\
\hline
\end{tabular}

Table 9 Convergence rates for $p=1.9$ in the scheme $\mathbf{U V}$

\begin{tabular}{lllllllll}
\hline$m \times m$ & $\left\|e_{u}^{n}\right\|_{l^{\infty}\left(L^{2}\right)}$ & Order & $\left\|e_{u}^{n}\right\|_{l^{2}\left(H^{1}\right)}$ & Order & $\left\|e_{v}^{n}\right\|_{l^{\infty}\left(L^{2}\right)}$ & Order & $\left\|e_{v}^{n}\right\|_{l^{2}\left(H^{1}\right)}$ & Order \\
\hline $36 \times 36$ & $5.0376 \mathrm{e}-03$ & - & $1.1650 \mathrm{e}-01$ & - & $4.9868 \mathrm{e}-03$ & - & $1.2842 \mathrm{e}-01$ & - \\
$44 \times 44$ & $3.3664 \mathrm{e}-03$ & 2.0087 & $9.5383 \mathrm{e}-02$ & 0.9966 & $3.3489 \mathrm{e}-03$ & 1.9841 & $1.0517 \mathrm{e}-01$ & 0.9951 \\
$52 \times 52$ & $2.4011 \mathrm{e}-03$ & 2.0227 & $8.0741 \mathrm{e}-02$ & 0.9976 & $2.4036 \mathrm{e}-03$ & 1.9853 & $8.9042 \mathrm{e}-02$ & 0.9966 \\
$60 \times 60$ & $1.7941 \mathrm{e}-03$ & 2.0367 & $6.9993 \mathrm{e}-02$ & 0.9982 & $1.8093 \mathrm{e}-03$ & 1.9849 & $7.7198 \mathrm{e}-02$ & 0.9974 \\
\hline
\end{tabular}

\section{Conclusions}

In this paper, the existence of global in time weak solutions for the chemorepulsion with $p$-power production model (3) and satisfying the energy inequality (8) has been proved in the 3D case, which are regular and unique in the $2 \mathrm{D}$ and $1 \mathrm{D}$ cases.

In addition, two new mass-conservative, unconditionally energy-stable and approximated positive fully discrete FE schemes for model (3), namely $\mathbf{U V} \varepsilon$ and $\mathbf{U S} \varepsilon$ have been developed. From the theoretical point of view, the following statements have been deduced:

(i) The solvability of both schemes.

(ii) The scheme $\mathbf{U V} \varepsilon$ is energy-stable with respect to the modified energy $\mathcal{E}_{\varepsilon}^{h}(u, v)$ (given in (59)), under the right-angled constraint $(\mathbf{H})$; while the scheme $\mathbf{U S} \varepsilon$ is unconditionally energy-stable with respect to the modified energy $\mathcal{E}_{\varepsilon}^{h}(u, \sigma)$ (given in (77)), without this restriction $(\mathbf{H})$ on the mesh.

(iii) It is not clear how to prove the energy-stability of the scheme UV (see Remark 5.1). 
(iv) In the schemes $\mathbf{U V} \varepsilon$ and $\mathbf{U S} \varepsilon$ there is a control for $\Pi^{h}\left(u_{\varepsilon-}^{n}\right)$ in $L^{2}$-norm, which tends to 0 as $\varepsilon \rightarrow 0$. This allows to conclude the non negativity of the solution $u_{\varepsilon}^{n}$ in the limit as $\varepsilon \rightarrow 0$.

On the other hand, from the numerical simulations, the following deductions can be made:

(i) The three schemes have decreasing in time energy $\mathcal{E}_{e}(u, v)$, independently of $\varepsilon$.

(ii) All the schemes show $R E_{e}^{n}>0$ for some $t_{n} \geq 0$; reaching highest values the scheme $\mathbf{U V}$, and the smallest values the scheme $\mathbf{U S} \varepsilon$. Moreover, scheme $\mathbf{U V} \varepsilon$ introduces lower numerical source than the scheme $\mathbf{U V}$, and lower numerical dissipation than scheme $\mathbf{U S} \varepsilon$.

(iii) Both schemes $\mathbf{U V} \varepsilon$ and $\mathbf{U S} \varepsilon$ satisfying that $\min _{\bar{\Omega} \times[0, T]} u_{\varepsilon}^{n} \rightarrow 0$ as $\varepsilon \rightarrow 0$.

(iv) All the schemes have optimal order of convergence in space, independent of the $p$-values.

\section{Appendix. Proof of Lemma 4.4}

The proof follows the ideas of [3, Lemma 2.1], with some modifications. For simplicity in the notation, we will prove (54) in the 1-dimensional case, but this proof can be extended to dimensions 2 and 3 as in [3, Lemma 2.1]. Observe that, from (52)

$$
\begin{aligned}
\left\|\left.\left(\Lambda_{\varepsilon}^{2}\left(u_{1}^{h}\right)-\Lambda_{\varepsilon}^{2}\left(u_{2}^{h}\right)\right)\right|_{K}\right\| \leq & \left.\left|\left(\Lambda_{\varepsilon}^{2}\left(u_{1}^{h}\right)-\Lambda_{\varepsilon}^{2}\left(u_{1,2}^{h}\right)\right)\right|_{K}|+|\left(\Lambda_{\varepsilon}^{2}\left(u_{1,2}^{h}\right)-\Lambda_{\varepsilon}^{2}\left(u_{2}^{h}\right)\right)\right|_{K} \mid \\
= & (p-1)\left|\frac{F_{\varepsilon}^{\prime}\left(\mu_{11}\right)}{F_{\varepsilon}^{\prime \prime}\left(\mu_{12}\right)}-\frac{F_{\varepsilon}^{\prime}\left(\xi_{1}\right)}{F_{\varepsilon}^{\prime \prime}\left(\xi_{2}\right)}\right| \\
& +(p-1)\left|\frac{F_{\varepsilon}^{\prime}\left(\xi_{1}\right)}{F_{\varepsilon}^{\prime \prime}\left(\xi_{2}\right)}-\frac{F_{\varepsilon}^{\prime}\left(\mu_{21}\right)}{F_{\varepsilon}^{\prime \prime}\left(\mu_{22}\right)}\right|
\end{aligned}
$$

where $u_{1,2}^{h} \in \mathbb{P}_{1}(K)$ with $u_{1,2}^{h}\left(\mathbf{a}_{0}^{K}\right)=u_{2}^{h}\left(\mathbf{a}_{0}^{K}\right)$ and $u_{1,2}^{h}\left(\mathbf{a}_{1}^{K}\right)=u_{1}^{h}\left(\mathbf{a}_{1}^{K}\right), \mu_{1 i}(i=1,2)$ lie between $u_{1}^{h}\left(\mathbf{a}_{0}^{K}\right)$ and $u_{1}^{h}\left(\mathbf{a}_{1}^{K}\right), \mu_{2 i}(i=1,2)$ lie between $u_{2}^{h}\left(\mathbf{a}_{0}^{K}\right)$ and $u_{2}^{h}\left(\mathbf{a}_{1}^{K}\right)$, and $\xi_{i}(i=1,2)$ lie between $u_{1}^{h}\left(\mathbf{a}_{1}^{K}\right)$ and $u_{2}^{h}\left(\mathbf{a}_{0}^{K}\right)$. Then, first we will show that

$$
(p-1)\left|\frac{F_{\varepsilon}^{\prime}\left(\mu_{11}\right)}{F_{\varepsilon}^{\prime \prime}\left(\mu_{12}\right)}-\frac{F_{\varepsilon}^{\prime}\left(\xi_{1}\right)}{F_{\varepsilon}^{\prime \prime}\left(\xi_{2}\right)}\right| \leq 3 \varepsilon^{2(p-2)} \max \left\{1,(p-1) \varepsilon^{2(p-2)}\right\}\left|u_{1}^{h}\left(\mathbf{a}_{0}^{K}\right)-u_{2}^{h}\left(\mathbf{a}_{0}^{K}\right)\right|,
$$

for $u_{1}^{h}\left(\mathbf{a}_{0}^{K}\right) \neq u_{2}^{h}\left(\mathbf{a}_{0}^{K}\right)$, because the case $u_{1}^{h}\left(\mathbf{a}_{0}^{K}\right)=u_{2}^{h}\left(\mathbf{a}_{0}^{K}\right)$ is trivially true. With this aim, we consider $\gamma_{i}(i=1,2)$ lying between $u_{1}^{h}\left(\mathbf{a}_{0}^{K}\right)$ and $u_{2}^{h}\left(\mathbf{a}_{0}^{K}\right)$ such that

$$
F_{\varepsilon}^{\prime}\left(\gamma_{1}\right)=\frac{F_{\varepsilon}\left(u_{2}^{h}\left(\mathbf{a}_{0}^{K}\right)\right)-F_{\varepsilon}\left(u_{1}^{h}\left(\mathbf{a}_{0}^{K}\right)\right)}{u_{2}^{h}\left(\mathbf{a}_{0}^{K}\right)-u_{1}^{h}\left(\mathbf{a}_{0}^{K}\right)} \text { and } \quad F_{\varepsilon}^{\prime \prime}\left(\gamma_{2}\right)=\frac{F_{\varepsilon}^{\prime}\left(u_{2}^{h}\left(\mathbf{a}_{0}^{K}\right)\right)-F_{\varepsilon}^{\prime}\left(u_{1}^{h}\left(\mathbf{a}_{0}^{K}\right)\right)}{u_{2}^{h}\left(\mathbf{a}_{0}^{K}\right)-u_{1}^{h}\left(\mathbf{a}_{0}^{K}\right)}
$$


and therefore, from the definitions of $\xi_{i}, \gamma_{i}$ and $\mu_{1 i}, i=1,2$, given after (82) and (84), we deduce

$$
\begin{aligned}
& \left(u_{2}^{h}\left(\mathbf{a}_{0}^{K}\right)-u_{1}^{h}\left(\mathbf{a}_{0}^{K}\right)\right) F_{\varepsilon}^{\prime}\left(\gamma_{1}\right)=\left(u_{2}^{h}\left(\mathbf{a}_{0}^{K}\right)-u_{1}^{h}\left(\mathbf{a}_{1}^{K}\right)\right) F_{\varepsilon}^{\prime}\left(\xi_{1}\right)+\left(u_{1}^{h}\left(\mathbf{a}_{1}^{K}\right)-u_{1}^{h}\left(\mathbf{a}_{0}^{K}\right)\right) F_{\varepsilon}^{\prime}\left(\mu_{11}\right), \\
& \left(u_{2}^{h}\left(\mathbf{a}_{0}^{K}\right)-u_{1}^{h}\left(\mathbf{a}_{0}^{K}\right)\right) F_{\varepsilon}^{\prime \prime}\left(\gamma_{2}\right)=\left(u_{2}^{h}\left(\mathbf{a}_{0}^{K}\right)-u_{1}^{h}\left(\mathbf{a}_{1}^{K}\right)\right) F_{\varepsilon}^{\prime \prime}\left(\xi_{2}\right)+\left(u_{1}^{h}\left(\mathbf{a}_{1}^{K}\right)-u_{1}^{h}\left(\mathbf{a}_{0}^{K}\right)\right) F_{\varepsilon}^{\prime \prime}\left(\mu_{12}\right) .
\end{aligned}
$$

Then, for $u_{2}^{h}\left(\mathbf{a}_{0}^{K}\right), u_{1}^{h}\left(\mathbf{a}_{0}^{K}\right)$ and $u_{1}^{h}\left(\mathbf{a}_{1}^{K}\right)$, there are only 3 options: (1) $u_{1}^{h}\left(\mathbf{a}_{1}^{K}\right)$ lies between $u_{2}^{h}\left(\mathbf{a}_{0}^{K}\right)$ and $u_{1}^{h}\left(\mathbf{a}_{0}^{K}\right)$; (ii) $u_{2}^{h}\left(\mathbf{a}_{0}^{K}\right)$ lies between $u_{1}^{h}\left(\mathbf{a}_{1}^{K}\right)$ and $u_{1}^{h}\left(\mathbf{a}_{0}^{K}\right)$; and (iii) $u_{1}^{h}\left(\mathbf{a}_{0}^{K}\right)$ lies between $u_{1}^{h}\left(\mathbf{a}_{1}^{K}\right)$ and $u_{2}^{h}\left(\mathbf{a}_{0}^{K}\right)$.

Notice that from $(43)$ and $(44)$, we have that $F_{\varepsilon}^{\prime}$ and $(p-1) \frac{F_{\varepsilon}^{\prime}}{F_{\varepsilon}^{\prime \prime}}$ are globally Lipschitz functions with constants $\varepsilon^{p-2}$ and 1 respectively, and $\frac{1}{\left|F_{\varepsilon}^{\prime \prime}\right|} \leq \varepsilon^{p-2}$. Then, in case (i), taking into account that all intermediate values $\mu_{1 i}, \gamma_{i}, \xi_{i}(i=1,2)$ lie between $u_{2}^{h}\left(\mathbf{a}_{0}^{K}\right)$ and $u_{1}^{h}\left(\mathbf{a}_{0}^{K}\right)$, we have

$$
\begin{aligned}
& (p-1)\left|\frac{F_{\varepsilon}^{\prime}\left(\mu_{11}\right)}{F_{\varepsilon}^{\prime \prime}\left(\mu_{12}\right)}-\frac{F_{\varepsilon}^{\prime}\left(\xi_{1}\right)}{F_{\varepsilon}^{\prime \prime}\left(\xi_{2}\right)}\right| \leq(p-1)\left|\frac{F_{\varepsilon}^{\prime}\left(\mu_{11}\right)-F_{\varepsilon}^{\prime}\left(\mu_{12}\right)}{F_{\varepsilon}^{\prime \prime}\left(\mu_{12}\right)}\right| \\
& +(p-1)\left|\frac{F_{\varepsilon}^{\prime}\left(\mu_{12}\right)}{F_{\varepsilon}^{\prime \prime}\left(\mu_{12}\right)}-\frac{F_{\varepsilon}^{\prime}\left(\xi_{2}\right)}{F_{\varepsilon}^{\prime \prime}\left(\xi_{2}\right)}\right|+(p-1)\left|\frac{F_{\varepsilon}^{\prime}\left(\xi_{1}\right)-F_{\varepsilon}^{\prime}\left(\xi_{2}\right)}{F_{\varepsilon}^{\prime \prime}\left(\xi_{2}\right)}\right| \\
\leq & (p-1) \varepsilon^{2(p-2)}\left|\mu_{11}-\mu_{12}\right|+\left|\mu_{12}-\xi_{2}\right|+(p-1) \varepsilon^{2(p-2)}\left|\xi_{1}-\xi_{2}\right| \\
\leq & 3 \max \left\{1,(p-1) \varepsilon^{2(p-2)}\right\}\left|u_{1}^{h}\left(\mathbf{a}_{0}^{K}\right)-u_{2}^{h}\left(\mathbf{a}_{0}^{K}\right)\right| .
\end{aligned}
$$

In case (ii), all intermediate values $\mu_{1 i}, \gamma_{i}, \xi_{i}(i=1,2)$ lie between $u_{1}^{h}\left(\mathbf{a}_{1}^{K}\right)$ and $u_{1}^{h}\left(\mathbf{a}_{0}^{K}\right)$, and from (85) and (86) by eliminating the term $\left(u_{2}^{h}\left(\mathbf{a}_{0}^{K}\right)-u_{1}^{h}\left(\mathbf{a}_{1}^{K}\right)\right)$, we have the equality

$$
\begin{gathered}
\left(u_{1}^{h}\left(\mathbf{a}_{1}^{K}\right)-u_{1}^{h}\left(\mathbf{a}_{0}^{K}\right)\right)\left[\frac{F_{\varepsilon}^{\prime}\left(\xi_{1}\right)}{F_{\varepsilon}^{\prime \prime}\left(\xi_{2}\right)}-\frac{F_{\varepsilon}^{\prime}\left(\mu_{11}\right)}{F_{\varepsilon}^{\prime \prime}\left(\mu_{12}\right)}\right]=\left(u_{2}^{h}\left(\mathbf{a}_{0}^{K}\right)\right. \\
\left.-u_{1}^{h}\left(\mathbf{a}_{0}^{K}\right)\right) \frac{F_{\varepsilon}^{\prime \prime}\left(\gamma_{2}\right)}{F_{\varepsilon}^{\prime \prime}\left(\mu_{12}\right)}\left[\frac{F_{\varepsilon}^{\prime}\left(\xi_{1}\right)}{F_{\varepsilon}^{\prime \prime}\left(\xi_{2}\right)}-\frac{F_{\varepsilon}^{\prime}\left(\gamma_{1}\right)}{F_{\varepsilon}^{\prime \prime}\left(\gamma_{2}\right)}\right]
\end{gathered}
$$

from which, bounding the term $\left|\frac{F_{\varepsilon}^{\prime}\left(\xi_{1}\right)}{F_{\varepsilon}^{\prime \prime}\left(\xi_{2}\right)}-\frac{F_{\varepsilon}^{\prime}\left(\gamma_{1}\right)}{F_{\varepsilon}^{\prime \prime}\left(\gamma_{2}\right)}\right|$ as in (87), we obtain

$$
\begin{aligned}
& \left.(p-1) \mid u_{1}^{h}\left(\mathbf{a}_{1}^{K}\right)-u_{1}^{h}\left(\mathbf{a}_{0}^{K}\right)\right)|| \frac{F_{\varepsilon}^{\prime}\left(\mu_{11}\right)}{F_{\varepsilon}^{\prime \prime}\left(\mu_{12}\right)}-\frac{F_{\varepsilon}^{\prime}\left(\xi_{1}\right)}{F_{\varepsilon}^{\prime \prime}\left(\xi_{2}\right)} \mid \\
& \left.\leq \varepsilon^{2(p-2)} 3 \max \left\{1,(p-1) \varepsilon^{2(p-2)}\right\}\left|u_{1}^{h}\left(\mathbf{a}_{0}^{K}\right)-u_{2}^{h}\left(\mathbf{a}_{0}^{K}\right)\right| \mid u_{1}^{h}\left(\mathbf{a}_{1}^{K}\right)-u_{1}^{h}\left(\mathbf{a}_{0}^{K}\right)\right) \mid,
\end{aligned}
$$

and therefore, dividing by $\left.\mid u_{1}^{h}\left(\mathbf{a}_{1}^{K}\right)-u_{1}^{h}\left(\mathbf{a}_{0}^{K}\right)\right) \mid$ we arrive at

$(p-1)\left|\frac{F_{\varepsilon}^{\prime}\left(\mu_{11}\right)}{F_{\varepsilon}^{\prime \prime}\left(\mu_{12}\right)}-\frac{F_{\varepsilon}^{\prime}\left(\xi_{1}\right)}{F_{\varepsilon}^{\prime \prime}\left(\xi_{2}\right)}\right| \leq 3 \varepsilon^{2(p-2)} \max \left\{1,(p-1) \varepsilon^{2(p-2)}\right\}\left|u_{1}^{h}\left(\mathbf{a}_{0}^{K}\right)-u_{2}^{h}\left(\mathbf{a}_{0}^{K}\right)\right|$. 
In case (iii), by arguing analogously to case (ii), from (85) and (86) we have

$$
\begin{gathered}
\left(u_{1}^{h}\left(\mathbf{a}_{1}^{K}\right)-u_{2}^{h}\left(\mathbf{a}_{0}^{K}\right)\right)\left[\frac{F_{\varepsilon}^{\prime}\left(\xi_{1}\right)}{F_{\varepsilon}^{\prime \prime}\left(\xi_{2}\right)}-\frac{F_{\varepsilon}^{\prime}\left(\mu_{11}\right)}{F_{\varepsilon}^{\prime \prime}\left(\mu_{12}\right)}\right]=\left(u_{2}^{h}\left(\mathbf{a}_{0}^{K}\right)\right. \\
\left.-u_{1}^{h}\left(\mathbf{a}_{0}^{K}\right)\right) \frac{F_{\varepsilon}^{\prime \prime}\left(\gamma_{2}\right)}{F_{\varepsilon}^{\prime \prime}\left(\xi_{2}\right)}\left[\frac{F_{\varepsilon}^{\prime}\left(\gamma_{1}\right)}{F_{\varepsilon}^{\prime \prime}\left(\gamma_{2}\right)}-\frac{F_{\varepsilon}^{\prime}\left(\mu_{11}\right)}{F_{\varepsilon}^{\prime \prime}\left(\mu_{12}\right)}\right],
\end{gathered}
$$

which implies (88). Therefore, we have proved (83). Analogously, we can prove that $(p-1)\left|\frac{F_{\varepsilon}^{\prime}\left(\xi_{1}\right)}{F_{\varepsilon}^{\prime \prime}\left(\xi_{2}\right)}-\frac{F_{\varepsilon}^{\prime}\left(\mu_{21}\right)}{F_{\varepsilon}^{\prime \prime}\left(\mu_{22}\right)}\right| \leq 3 \varepsilon^{2(p-2)} \max \left\{1,(p-1) \varepsilon^{2(p-2)}\right\}\left|u_{1}^{h}\left(\mathbf{a}_{1}^{K}\right)-u_{2}^{h}\left(\mathbf{a}_{1}^{K}\right)\right|$.

Thus, from (82), (83) and (89) we conclude (54).

Acknowledgements The authors would like to thank the anonymous referees for their valuable comments.

Funding Open Access funding provided thanks to the CRUE-CSIC agreement with Springer Nature. The authors have been partially supported by MINECO grant MTM2015-69875-P (Ministerio de Economía y Competitividad, Spain) with the participation of FEDER. The first and second authors have also been supported by Grant PGC2018-098308-B-I00 by MCIN/AEI/ 10.13039/501100011033 and by ERDF A way of making Europe; and the third author has also been supported by Vicerrectoría de Investigación y Extensión of Universidad Industrial de Santander.

\section{Declarations}

Conflict of interest The authors declare no competing interests.

Open Access This article is licensed under a Creative Commons Attribution 4.0 International License, which permits use, sharing, adaptation, distribution and reproduction in any medium or format, as long as you give appropriate credit to the original author(s) and the source, provide a link to the Creative Commons licence, and indicate if changes were made. The images or other third party material in this article are included in the article's Creative Commons licence, unless indicated otherwise in a credit line to the material. If material is not included in the article's Creative Commons licence and your intended use is not permitted by statutory regulation or exceeds the permitted use, you will need to obtain permission directly from the copyright holder. To view a copy of this licence, visit http://creativecommons.org/licenses/by/4.0/.

\section{References}

1. Amrouche, C., Seloula, N.E.H.: $L^{p}$-theory for vector potentials and Sobolev's inequalities for vector fields: application to the Stokes equations with pressure boundary conditions. Math. Models Methods Appl. Sci. 23(1), 37-92 (2013)

2. Barrett, J., Blowey, J.: Finite element approximation of a nonlinear cross-diffusion population model. Numer. Math. 98(2), 195-221 (2004)

3. Barrett, J., Nürnberg, R.: Finite-element approximation of a nonlinear degenerate parabolic system describing bacterial pattern formation. Interfaces Free Bound. 4(3), 277-307 (2002)

4. Becker, R., Feng, X., Prohl, A.: Finite element approximations of the Ericksen-Leslie model for nematic liquid crystal flow. SIAM J. Numer. Anal. 46, 1704-1731 (2008) 
5. Bellomo, N., Bellouquid, A., Tao, Y., Winkler, M.: Toward a mathematical theory of Keller-Segel models of pattern formation in biological tissues. Math. Models Methods Appl. Sci. 25(9), 1663-1763 (2015)

6. Bessemoulin-Chatard, M., Jüngel, A.: A finite volume scheme for a Keller-Segel model with additional cross-diffusion. IMA J. Numer. Anal. 34(1), 96-122 (2014)

7. Chamoun, G., Saad, M., Talhouk, R.: Monotone combined edge finite volume-finite element scheme for anisotropic Keller-Segel model. Numer. Methods Partial Differ. Equ. 30(3), 1030-1065 (2014)

8. Chamoun, G., Saad, M., Talhouk, R.: Numerical analysis of a chemotaxis-swimming bacteria model on a general triangular mesh. Appl. Numer. Math. 127, 324-348 (2018)

9. Cieslak, T., Laurencot, P., Morales-Rodrigo, C.: Global Existence and Convergence to Steady States in a Chemorepulsion System. Parabolic and Navier-Stokes Equations. Part 1, 105-117, Banach Center Publ., 81 Part 1, Polish Acad. Sci. Inst. Math., Warsaw (2008)

10. De Leenheer, P., Gopalakrishnan, J., Zuhr, E.: Nonnegativity of exact and numerical solutions of some chemotactic models. Comput. Math. Appl. 66(3), 356-375 (2013)

11. Epshteyn, Y., Izmirlioglu, A.: Fully discrete analysis of a discontinuous finite element method for the Keller-Segel chemotaxis model. J. Sci. Comput. 40(1-3), 211-256 (2009)

12. Feireisl, E., Novotna, A.: Singular Limits in Thermodynamics of Viscous Fluids. Advances in Mathematical Fluid Mechanics. Basel, BirkhAuser (2009)

13. Filbet, F.: A finite volume scheme for the Patlak-Keller-Segel chemotaxis model. Numer. Math. 104(4), 457-488 (2006)

14. Freitag, M.: Global existence and boundedness in a chemorepulsion system with superlinear diffusion. Discret. Contin. Dyn. Syst. 38(11), 5943-5961 (2018)

15. Grisvard, P.: Elliptic Problems in Nonsmooth Domains. Pitman Advanced Publishing Program, Boston (1985)

16. Grün, G., Rumpf, M.: Nonnegativity preserving convergent schemes for the thin film equation. Numer. Math. 87, 113-152 (2000)

17. Guillén-González, F., Rodríguez-Bellido, M.A., Rueda-Gómez, D.A.: Study of a chemo-repulsion model with quadratic production. Part I: Analysis of the continuous problem and time-discrete numerical schemes. Comput. Math. Appl. 80, 692-713 (2020)

18. Guillén-González, F., Rodríguez-Bellido, M.A., Rueda-Gómez, D.A.: Study of a chemo-repulsion model with quadratic production. Part II: Analysis of an unconditionally energy-stable fully discrete scheme. Comput. Math. Appl. 80, 636-652 (2020)

19. Guillén-González, F., Rodríguez-Bellido, M.A., Rueda-Gómez, D.A.: Comparison of two finite element schemes for a chemo-repulsion system with quadratic production. (Submitted). arXiv:1805.00962 [math.NA]

20. Guillén-González, F., Rodríguez-Bellido, M.A., Rueda-Gómez, D.A.: Unconditionally energy stable fully discrete schemes for a chemo-repulsion model. Math. Comput. 88(319), 2069-2099 (2019)

21. He, Y., Li, K.: Asymptotic behavior and time discretization analysis for the non-stationary NavierStokes problem. Numer. Math. 98(4), 647-673 (2004)

22. Ibrahim, M., Saad, M.: On the efficacy of a control volume finite element method for the capture of patterns for a volume-filling chemotaxis model. Comput. Math. Appl. 68(9), 1032-1051 (2014)

23. Lai, Y., Xiao, Y.: Existence and asymptotic behavior of global solutions to chemorepulsion systems with nonlinear sensitivity. Electron. J. Differential Equations, Paper No. 254, pp. 9 (2017)

24. Lions, J.L., Magenes, E.: Problèmes Aux Limites Non Homogg̀Nes Et Applications, vol. 1. Travaux Et Recherches MathéMatiques, No. 17 Dunod, Paris (1968)

25. Marrocco, A.: Numerical simulation of chemotactic bacteria aggregation via mixed finite elements. m2AN Math. Model. Numer. Anal. 37(4), 617-630 (2003)

26. Necas, J.: Les Méthodes Directes En Théorie Des Equations Elliptiques. Editeurs Academia, Prague (1967)

27. Saito, N.: Conservative upwind finite-element method for a simplified Keller-Segel system modelling chemotaxis. IMA J. Numer. Anal. 27(2), 332-365 (2007)

28. Saito, N.: Error analysis of a conservative finite-element approximation for the Keller-Segel system of chemotaxis. Commun. Pure Appl. Anal. 11(1), 339-364 (2012)

29. Simon, J.: Compact sets in the space $l^{p}(0, t ; b)$. Ann. Mat. Pura Appl. 146(4), 65-96 (1987)

30. Tao, Y.: Global dynamics in a higher-dimensional repulsion chemotaxis model with nonlinear sensitivity. Discret. Contin. Dyn. Syst. Ser. B 18(10), 2705-2722 (2013) 
31. Tello, J., Wrzosek, D.: Inter-species competition and chemorepulsion. J. Math. Anal. Appl. 459(2), 1233-1250 (2018)

32. Winkler, M.: A critical blow-up exponent in a chemotaxis system with nonlinear signal production. Nonlinearity 31(5), 2031-2056 (2018)

33. Zhang, J., Zhu, J., Zhang, R.: Characteristic splitting mixed finite element analysis of Keller-Segel chemotaxis models. Appl. Math. Comput. 278, 33-44 (2016)

34. Zhou, G., Saito, N.: Finite volume methods for a Keller-Segel system: discrete energy, error estimates and numerical blow-up analysis. Numer. Math. 135(1), 265-311 (2017)

Publisher's note Springer Nature remains neutral with regard to jurisdictional claims in published maps and institutional affiliations. 


\title{
Affiliations
}

\section{F. Guillén-González ${ }^{1}$ (D) - M. A. Rodríguez-Bellido ${ }^{1}$. D. A. Rueda-Gómez ${ }^{2}$}

\author{
M. A. Rodríguez-Bellido \\ angeles@us.es \\ D. A. Rueda-Gómez \\ diaruego@uis.edu.co \\ 1 Dpto. Ecuaciones Diferenciales y Análisis Numérico and IMUS, Universidad de Sevilla, \\ Facultad de Matemáticas, C/ Tarfia, S/N, 41012 Sevilla, Spain \\ 2 Escuela de Matemáticas, Universidad Industrial de Santander, \\ A.A. 678 , Bucaramanga, Colombia
}

